\title{
IOPB COLUMN
}

\section{Edited by Karol Marhold \& Ilse Breitwieser}

\section{IAPT/IOPB chromosome data 16}

\section{Edited by Karol Marhold}

Laura Chalup ${ }^{1 *}$ \& Guillermo Seijo ${ }^{1,2}$

1 Instituto de Botánica del Nordeste (UNNE-CONICET), Facultad de Ciencias Agrarias, Sgto. Cabral 2131, 3400 Corrientes, Argentina

2 Facultad de Ciencias Exactas y Naturales y Agrimensura, Universidad Nacional del Nordeste, Avenida Libertad 5500 3400 Corrientes, Argentina

* Author for correspondence: lchalup@agr.unne.edu.ar

This work was supported by Agencia Nacional de Promoción Científica y Tecnológica, Argentina PICTO-UNNE 090. Laura Chalup is fellow of Consejo Nacional de Investigaciones Científicas y Técnicas (CONICET), Argentina.

All numbers CHN; collectors: $A=\mathrm{R}$. Almada, $B=\mathrm{H}$. Bogado, $C h=\mathrm{L}$. Chalup, $D=\mathrm{M}$. Dematteis, $L=\mathrm{G}$. Lavia, $M a=\mathrm{C}$. Mackluf, $G$ $=\mathrm{A}$. González, $M e=\mathrm{W}$. Medina, $M t=\mathrm{E}$. Meza Torres, $R=\mathrm{H}$. Roig, $S=$ G. Seijo, $S c=$ A. Schinini, $S n=$ V. Solís Neffa

\section{FABACEAE}

Lathyrus crassipes Gillies ex Hook. \& Arn., $2 n=14$; Argentina, Corrientes, $S, L, R \& B 2349$ (CTES, G, U).

Lathyrus latifolius L., $2 n=14$; Argentina, Córdoba, Sn 1998 (CTES). $n=7$; Argentina, Río Negro, $S 1170$ (CTES, LIL, SPF).

Lathyrus macrostachys Vogel, $n=7$; Argentina, Misiones, $C h, S, S n$ \& $G 1$ (CTES).

Lathyrus magellanicus var. glaucescens Speg., $2 n=14$; Argentina, Chubut, $C h, M e, M a \& M t 19$ (CTES, MO, NY).

Lathyrus magellanicus Lam. var. magellanicus, $2 n=14$; Argentina, Río Negro, S 2307 (CTES, K, NY, SI).

Lathyrus magellanicus var. tucumanensis Burkart, $n=7$; Argentina, Jujuy, $S \& D 1109$ (CEN, CTES, LPB, MBM, NY).

Lathyrus multiceps Clos, $2 n=14$; Argentina, Neuquén, $S 1607$ (CTES, F, TEX). $n=7$; Argentina, Neuquén, $S 1194$ (CTES, K, MICH); $S 1653$ (ALCB, CTES, G, HUEFS, MBM).

Lathyrus nitens Vogel, $2 n=14$; Uruguay, Tacuarembó, $S$ \& Sn 3966 (CTES, UTEP).

Lathyrus paranensis Burkart, 2n=14; Uruguay, Rivera, $S \& S n 3954$ (ASU, BGE, CTES, FUEL, I, MBM); Uruguay, Colonia, $S \& S n$ 3984 (CTES).

Lathyrus pubescens Hook. \& Arn., $2 n=14$; Uruguay, Rivera, $S \& S n$ 3941 (CTES, FUEL, IAC, ICN, MBM, MO); Uruguay, San José, $S$ \& Sn 3972 (CEN, CTES); Argentina, Buenos Aires, S 2332 (CTES). $n=7$; Argentina, Tucumán, $S \& D 1086$ (CTES, CUVC, HUEFS, MBM, MICH, NY); Argentina, San Luis, S 2059 (CTES); Bolivia, Santa Cruz, S, Sn, Sc \& A 3082 (CTES).
DOI: http://dx.doi.org/10.12705/626.41

\section{Neli H. Grozeva}

Department of Biology and Aquaculture, Agricultural Faculty, Trakia University,6000 Stara Zagora,Bulgaria; grozeva@uni-sz.bg

All materials CHN, collected from Bulgaria, vouchers in SOM.

This study was supported by the Project Scientific Research Fund of Trakia University, Agriculture faculty.

\section{CHENOPODIACEAE}

Atriplex oblongifolia Waldst. \& Kit., $2 n=36 ;$ N.H. Grozeva 4058. Atriplex tatarica L., $2 n=18 ;$ N.H. Grozeva 4059.

Bassia hirsuta (L.) Asch., $2 n=18 ;$ N.H. Grozeva 4060.

Bassia laniflora (S.G. Gmel.) A.J. Scott, $2 n=18 ;$ N.H. Grozeva 4062.

Chenopodium album L., $2 n=54 ;$ N.H. Grozeva 4061.

Chenopodium chenopodioides (L.) Aellen, $2 n=18$; N.H. Grozeva 4063.

Chenopodium polyspermum L., $2 n=18 ;$ N.H. Grozeva 4064. Chenopodium rubrum L., $2 n=18 ;$ N.H. Grozeva 4067.

Chenopodium strictum Roth, $2 n=36 ;$ N.H. Grozeva 4065 .

Chenopodium urbicum L., $2 n=18 ;$ N.H. Grozeva 4066.

Corispermum nitidum Kit. ex Schult., $2 n=18 ;$ N.H. Grozeva 4068.

Dysphania pumilio (R. Br.) Mosyakin \& Clemants, $2 n=18 ; N . H$. Grozeva 4069

Salicornia europaea L., $2 n=18 ;$ N.H. Grozeva 4070.

Salsola tragus L., $2 n=36 ;$ N.H. Grozeva 4071.

Denis A. Krivenko, ${ }^{1 *}$ Violetta V. Kotseruba, ${ }^{2}$ Sergey G. Kazanovsky, ${ }^{1}$ Alla V. Verkhozina ${ }^{1} \&$ Olga D. Chernova ${ }^{3}$

1 Institute of Plant Physiology \& Biochemistry, Siberian Branch of the Russian Academy of Sciences, 132 Lermontov Str., 664033, Irkutsk, Russia

2 Komarov Botanical Institute of the Russian Academy of Sciences, 2 Prof. Popov Str., 197376, St.-Petersburg, Russia

3 Zabaikalsky State Humanitarian Pedagogical University named after N. Chernishevsky, 129 Babushkina Str., 672007, Chita, Russia

* Author for correspondence: krivenko.irk@gmail.com

All materials CHN; collectors: $A V=$ A.V. Verkhozina, $D K=$ D.A. Krivenko, $O C=$ O.D. Chernova, $S K=$ S.G. Kazanovsky, $V K=$ V.V. Kotseruba.

This study was supported by grants no. 11-04-00240-a, 1104-01167-a, 12-04-01586-a, 12-04-31151 mol-a, 13-04-00351-a,

All materials for the chromosome column should be submitted electronically to: Karol Marhold, karol.marhold@savba.sk (Institute of Botany, Slovak Academy of Sciences, SK-845 23 Bratislava, Slovakia, and Department of Botany, Charles University, CZ 128-01 Prague, Czech Republic). The full version of this contribution is available in the online edition of TAXON appended to this article. The following citation format is recommended: Baltisberger, M. \& Voelger, M. 2006. Sternbergia sicula. In: Marhold, K. (ed.), IAPT/IOPB chromosome data 1. Taxon 55: 444, E2. 
13-04-01458-a, 13-04-01468-a from the Russian Fund for Basic Research (RFBR) and by Interdisciplinary integration project Nr. 17, from the Siberian Branch of the Russian Academy of Sciences.

\section{ACORACEAE}

Acorus calamus L., $2 n=24$; Russia, Republic of Buryatiya, AV 19864 (IRK).

\section{ADOXACEAE}

Adoxa moschatellina L., $2 n=36$; Russia, Republic of Altai, DK 29573 (IRK).

\section{APIACEAE}

Conium maculatum L., $2 n=22$; Russia, Novosibirskaya Oblast', $S K$ 26612 (IRK).

\section{ASCLEPIADACEAE}

Vincetoxicum sibiricum (L.) Decne., $2 n=22$; Russia, Zabaikal'skii Krai, Ye.A. Bondarevich 29562 (IRK).

\section{ASTERACEAE}

Artemisia halodendron Turcz. ex Besser, $2 n=36$; Mongolia, Dornod Aimak, A.A. Korobkov 09-92 (LE).

Artemisia insulana Krasch., $2 n=18$; Russia, Kamchatskii Krai, N.V. Sinel'nikova 99-203 (LE).

Artemisia tanacetifolia L., $2 n=54$; Russia, Altaiskii Krai, A.A. Korobkov 99-196 (LE).

Artemisia vulgaris L., $2 n=16$; Russia, Kemerovskaya Oblast', I.M Krasnoborov 00-111 (NS).

Cirsium esculentum (Siev.) C.A. Mey., $2 n=34$; Russia, Irkutskaya Oblast', $A V 19730$ (IRK).

Neopallasia pectinata (Pall.) Poljakov, $2 n=18$; Russia, Republic of Buryatiya, A.A. Korobkov 06-211 (LE).

Petasites frigidus (L.) Fr., $2 n=60$; Russia, Republic of Sakha (Yakutia), $D K 27259$ (IRK)

Senecio vulgaris L., $2 n=40$; Russia, Sverdlovskaya Oblast', O.S Dymshakova 21110 (IRK).

Sonchus asper (L.) Hill, $2 n=18$; Russia, Sverdlovskaya Oblast', O.S. Dymshakova 21105 (IRK).

Taraxacum officinale F.H. Wigg., $2 n=24$; Russia, Sverdlovskaya Oblast', O.S. Dymshakova 29603 (IRK).

Tragopogon orientalis L., $2 n=12$; Russia, Altaiskii Krai, DK 29637 (IRK).

Tragopogon trachycarpus S.A. Nikitin, $2 n=12$; Russia, Republic of Buryatiya, DK 28217 (IRK).

Tridactylina kirilowii (Turcz.) Sch. Bip., $2 n=18$; Russia, Irkutskaya Oblast', Yu.N. Pochinchik 24539 (IRK).

\section{BORAGINACEAE}

Craniospermum subvillosum Lehm., $2 n=24$; Russia, Republic of Buryatiya, DK 23123 (IRK).

Lappula tuvinica Ovczinnikova, $2 n=24$; Russia, Altaiskii Krai, $D K$ 29604 (IRK)

\section{BRASSICACEAE}

Alyssum tortuosum Waldst. \& Kit. ex Willd., $2 n=16$; Russia, Altaiskii Krai, DK 29633 (IRK).

Capsella bursa-pastoris (L.) Medik., $2 n=32$; Russia, Sverdlovskaya Oblast', O.S. Dymshakova 21108 (IRK).

Lepidium ruderale L., $2 n=16$; Russia, Irkutskaya Oblast', DK 29607 (IRK).

\section{CAPRIFOLIACEAE}

Linnaea borealis L., $2 n=32$; Russia, Republic of Buryatiya, $D K$ 23271 (IRK).

\section{CARYOPHYLLACEAE}

Stellaria media (L.) Vill., $2 n=$ ca. 40; Russia, Sverdlovskaya Oblast', O.S. Dymshakova 21109 (IRK).

\section{CHENOPODIACEAE}

Corispermum sibiricum Iljin, $2 n=18$; Russia, Republic of Sakha (Yakutia), DK 29606 (IRK).

\section{CLUSIACEAE}

Hypericum attenuatum Choisy, $2 n=16$; Russia, Irkutskaya Oblast', DK 29634 (IRK).

\section{CYPERACEAE}

Carex pamirica subsp. dichroa (Freyn) T.V. Egorova, $2 n=$ ca. 32-34; Russia, Republic of Buryatiya, DK 20658 (IRK).

EPHEDRACEAE

Ephedra monosperma C.A. Mey., $2 n=14$; Russia, Irkutskaya Oblast', T.V. Krivenko 29512 (IRK)

\section{FABACEAE}

Astragalus frigidus (L.) A. Gray, $2 n=16+0-1 \mathrm{~B}$; Russia, Irkutskaya Oblast', SK 24684 (IRK).

Lespedeza davurica (Laxm.) Schindl., $2 n=42$; Russia, Zabaikal'skii Krai, G.N. Zinov'yeva 29510 (IRK)

Melilotus albus Medik., $2 n=16$; Russia, Zabaikal'skii Krai, G.N. Zinov'yeva 29509 (IRK).

Oxytropis varlakovii Serg., $2 n=32$; Russia, Zabaikal'skii Krai, $O C$ 29507 (IRK)

Thermopsis lanceolata $\mathrm{R}$. Br., $2 n=18$; Russia, Zabaikal'skii Krai, G.N. Zinov'yeva 29503 (IRK), OC 29506 (IRK).

Vicia cracca L., $2 n=24$; Russia, Zabaikal'skii Krai, G.N. Zinov'yeva 29511 (IRK)

\section{IRIDACEAE}

Iris biglumis Vahl, $2 n=40$; Russia, Republic of Buryatiya, DK 19870 (IRK).

Iris laevigata Fisch. ex Fisch. \& C.A. Mey, $2 n=32$; Russia, Republic of Buryatiya, Yu.N. Pochinchik 24372 (IRK), AV 23573 (IRK).

Iris ruthenica Ker Gawl., 2n = ca. 80; Russia, Altaiskii Krai, DK 29574 (IRK); Russia, Republic of Altai, DK 29575 (IRK).

\section{LILIACEAE}

Tulipa biebersteiniana Schult. \& Schult. f., $2 n=24$; Russia, Republic of Bashkortostan, N.A. Kutlunina \& A.Yu. Belyaev 556693 (SVER); Russia, Orenburgskaya Oblast', N.A. Kutlunina \& A.Yu. Belyaev 06-05-06 (UFU), N.A. Kutlunina \& A.Yu. Belyaev 506719 (SVER), N.A. Kutlunina \& A.Yu. Belyaev 506722 (SVER); Russia, Volgogradskaya Oblast', N.A. Kutlunina \& M.I. Zerebtsova 18-04-07 (UFU), N.A. Kutlunina \& A.Yu. Belyaev 20-04-07 (UFU). 2n=36; Russia, Orenburgskaya Oblast', N.A. Kutlunina \& A.Yu. Belyaev 06-05-06 (UFU)

Tulipa patens Agardh. ex Schult. \& Schult. f., $2 n=24$; Russia, Orenburgskaya Oblast', N.A. Kutlunina \& A.Yu. Belyaev 06-05-06 (UFU). Tulipa riparia Knjaz., Kulikov \& E.G. Philippov, $2 n=36$; Russia, Chelyabinskaya Oblast', N.A. Kutlunina \& A.Yu. Belyaev 24-0506 (UFU), N.A. Kutlunina \& A.Yu. Belyaev 506711 (SVER), N.A. Kutlunina \& A.Yu. Belyaev 25-05-06 (UFU).

Tulipa scythica Klokov \& Zoz, $2 n=24$; Russia, Orenburgskaya Oblast', P.V. Kulikov 19-04-05 (UFU); Russia, Volgogradskaya Oblast', N.A. Kutlunina \& M.I. Zerebtsova 18-04-07 (UFU), N.A. Kutlunina \& M.I. Zerebtsova 20-04-07 (UFU).

\section{ONAGRACEAE}

Circaea alpina L., $2 n=22$; Russia, Republic of Altai, DK 29572 (IRK). 
Epilobium fastigiato-ramosum Nakai, $2 n=36$; Russia, Irkutskaya Oblast', AV 27773 (IRK).

\section{ORCHIDACEAE}

Cypripedium guttatum Sw., $2 n=20$; Russia, Republic of Buryatiya, $S K \& D K 19885$ (IRK).

Goodyera repens (L.) R. Br., $2 n=30$; Russia, Republic of Buryatiya, SK \& DK 19796 (IRK).

Gymnadenia conopsea (L.) R. Br., $2 n=40$; Russia, Republic of Buryatiya, $S K \& D K 20013$ (IRK).

Neottianthe cucullata (L.) Schltr., $2 n=42$; Russia, Republic of Buryatiya, $S K \& D K 20021$ (IRK).

\section{PLANTAGINACEAE}

Plantago depressa Willd., $2 n=12$; Russia, Zabaikal'skii Krai, $S K$ 20781 (IRK); Russia, Republic of Buryatiya, DK 20014 (IRK).

Plantago major L., $2 n=12$; Russia, Sverdlovskaya Oblast', O.S. Dymshakova 29598 (IRK).

\section{POACEAE}

Melica turczaninowiana Ohwi, $2 n=18$; Russia, Zabaikal'skii Krai, Ye.A. Bondarevich 22402 (IRK).

Milium alexeenkoi (Tzvelev) Tzvelev, $2 n=14$; Russia, Republic of Dagestan, M.P. Rayko \& VK 29597 (IRK, LE).

Phragmites flavescens (Custer) Hegetschw., $2 n=48$; Kazakhstan, Pavlodarskaya Oblast', DK 29642 (IRK).

Spodiopogon sibiricus Trin., $2 n=40$; Russia, Zabaikal'skii Krai, Ye.A. Bondarevich 22399 (IRK).

\section{POLEMONIACEAE}

Collomia linearis Nutt., $2 n=16$; Russia, Irkutskaya Oblast', N.V. Dorofeev 24475 (IRK).

Polemonium caeruleum L., $2 n=18$; Russia, Republic of Altai, $D K$ 29658 (IRK).

\section{RANUNCULACEAE}

Aconitum sukaczevii Steinb., $2 n=16$; Russia, Irkutskaya Oblast', $S K$ 19410 (IRK).

Ranunculus chinensis Bunge, $2 n=16+0-4 \mathrm{~B}$; Russia, Zabaikal'skii Krai, Ye.S. Prelovskaya \& S.G. Kazanovsky 20959 (IRK).

\section{ROSACEAE}

Coluria geoides (Pall.) Ledeb., $2 n=14$; Russia, Altaiskii Krai, $D K$ 29576 (IRK).

\section{SCROPHULARIACEAE}

Veronica prostrata L., $2 n=16$; Russia, Irkutskaya Oblast', $A V \&$ al. 27830 (IRK).

SOLANACEAE

Hyoscyamus niger L., $2 n=34$; Russia, Republic of Altai, DK 29628 (IRK).

\section{THYMELAEACEAE}

Daphne mezereum L., $2 n=18$; Russia, Republic of Buryatiya, $S K$ 21127 (IRK).

VIOLACEAE

Viola rupestris F.W. Schmidt, $2 n=20$; Russia, Irkutskaya Oblast', DK 24499 (IRK).

Viola selkirkii Pursh ex Goldie, $2 n=24$; Russia, Irkutskaya Oblast', SK \& DK 19469 (IRK).

\section{Maria N. Lomonosova}

Central Siberian Botanical Garden, Siberian Branch of the Russian Academy of Science, Novosibirsk 630090, Russia; mlomonosova@mail.ru

All materials CHN; collectors: $E N=$ Evgeniy G. Nikolin, $M L=$ Maria N. Lomonosova; vouchers in NS.

This research was supported by the Russian Foundation for Basic Research (grants no. 11-04-00123, 12-04-00746).

\section{AMARANTHACEAE}

Amaranthus retroflexus L., $2 n=34$; Russia, Kalmykia Republic, ML 937a.

\section{ASTERACEAE}

Carduus crispus L., $2 n=18$; Russia, Kalmykia Republic, ML 959a. Centaurea diffusa Lam., $2 n=18$; Russia, Kalmykia Republic, $M L$ 941a.

Lactuca serriola L., $2 n=18$; Russia, Novosibirskaya Oblast', $M L 925 a$.

Lactuca tatarica (L.) C.A. Mey., $2 n=18$; Russia, Kalmykia Republic, ML 952a, ML 961.

Saussurea amara (L.) DC., $2 n=26$; Russia, Novosibirskaya Oblast', ML $925 b$

Tragopogon podolicus (DC.) S.A. Nikitin, $2 n=12$; Russia, Novosibirskaya Oblast', $M L$ 924a, $M L$ 925c.

Xanthium strumarium L., $2 n=36$; Russia, Novosibirskaya Oblast', ML $926 a$.

\section{BRASSICACEAE}

Lepidium densiflorum Schrad., $2 n=16$; Russia, Sakha (Yakutia) Republic, $M L \& E N 859$.

Thlaspi arvense L., $2 n=14$; Russia, Yakutia Republic, $M L \&$ EN $791 a$.

\section{CARYOPHYLLACEAE}

Dianthus versicolor Fisch. \& Link, $2 n=60$; Russia, Sakha (Yakutia) Republic, $M L$ \& EN 793.

\section{CHENOPODIACEAE}

Atriplex micrantha C.A. Mey., $2 n=36$; Russia, Kalmykia Republic, ML 940, ML 949a.

Atriplex patens (Litv.) Iljin, $2 n=36$; Russia, Novosibirskaya Oblast', ML 922.

Atriplex prostrata Boucher \& DC., $2 n=18$; Russia, Kalmykia Republic, ML 954a, ML 959b.

Atriplex sagittata Borkh., $2 n=18$; Russia, Novosibirskaya Oblast', ML 924b.

Atriplex tatarica L., $2 n=18$; Russia, Kalmykia Republic, $M L 937 b$, ML $941 b$.

Bassia hyssopifolia (Pall.) Kuntze, $2 n=18$; Russia, Kalmykia Republic, ML 958a.

Bassia scoparia (L.) A.J. Scott, $2 n=18$; Russia, Kalmykia Republic, ML 958b; Russia, Khakasia Republic, F. Kudrov 963.

Caroxylon dendroides (Pall.) Tzvelev, $2 n=18$; Russia, Kalmykia Republic, $M L 947$.

Chenopodium acerifolium Andrz., $2 n=18$; Russia, Sakha (Yakutia) Republic, $M L \& E N 876$.

Chenopodium album L., $2 n=54$; Russia, Kalmykia Republic, $M L 949 a$.

Chenopodium borbasii Murr, $2 n=54$; Russia, Kalmykia Republic, ML 957.

Chenopodium ficifolium Sm., $2 n=18$; Russia, Sakha (Yakutia) Republic, $M L$ \& EN $791 b$.

Chenopodium strictum Roth, $2 n=36$; Russia, Novosibirskaya Oblast', ML 925d; Russia, Kalmykia Republic, ML 960.

Chenopodium suaecicum Murr, $2 n=18$; Russia, Novosibirsk, ML 965. 
Kali tragus (L.) Scop., $2 n=36$; Russia, Novosibirskaya Oblast', $M L$ 926b; Russia, Kalmykia Republic, ML 944, ML 958c.

Oxybasis chenopodioides (L.) S. Fuentes, Uotila \& Borsch, $2 n=18$; Russia, Kalmykia Republic, $M L 952 b$.

Oxybasis glauca (L.) S. Fuentes, Uotila \& Borsch, $2 n=18$; Russia, Novosibirskaya Oblast', $M L 925 e$.

Oxybasis urbica (L.) S. Fuentes, Uotila \& Borsch, $2 n=18$; Russia, Kalmykia Republic, $M L$ 944, $M L$ 949c.

Salicornia perennans Willd., $2 n=18$; Russia, Kalmykia Republic, $M L$ 952c; Russia, Sakha (Yakutia) Republic, $M L \& E N 783, M L \&$ EN 790, ML \& EN 796.

Salsola soda L., $2 n=18$; Russia, Kalmykia Republic, ML $938 a$.

Sedobassia sedoides (Pall.) Freitag \& G. Kadereit, $2 n=18$; Russia, Novosibirskaya Oblast', $M L 924 c$.

Suaeda acuminata (C.A. Mey.) Moq., $2 n=18$; Russia, Kalmykia Republic, ML 938b, ML 951.

Suaeda corniculata (C.A. Mey.) Bunge subsp. corniculata, $2 n=54$; Russia, Khakasia Republic, F. Kudrov 964a.

Suaeda salsa (L.) Pall., $2 n=36$; Russia, Kalmykia Republic, $M L$ 937c, ML 948, ML 954b, ML 958d, ML 959c; Russia, Volgogradskaya Oblast', $M L 962$.

Suaeda sibirica Lomon. \& Freitag, $2 n=72$; Russia, Khakasia Republic, F. Kudrov $964 b$.

\section{PLANTAGINACEAE}

Plantago cornuti Gouan, $2 n=12$; Russia, Novosibirskaya Oblast', ML $925 f$.

\section{ROSACEAE}

Potentilla paradoxa Nutt. ex Torr. \& Gray, $2 n=42$; Russia, Novosibirskaya Oblast', $M L 925 g$.

Nina S. Probatova, ${ }^{1 *}$ Sergey G. Kazanovsky, ${ }^{2}$ Elvira G. Rudyka, ${ }^{1}$ Vitaly P. Seledets ${ }^{3} \&$ Svetlana V. Ovchinnikova ${ }^{4}$

1 Institute of Biology \& Soil Science, Far East Branch of the Russian Academy of Sciences, 159 Stoletya Prospect, 690022, Vladivostok, Russia

2 Siberian Institute of Plant Physiology \& Biochemistry, Siberian Branch of the Russian Academy of Sciences, 132 Lermontov Str. 664033, Irkutsk, Russia

3 Pacific Institute of Geography, Far East Branch of the Russian Academy of Sciences, 7 Radio Str., 690041, Vladivostok, Russia

4 Central Siberian Botanical Garden, Siberian Branch of the Russian Academy of Sciences, 101 Zolotodolinskaya Str., 630090, Novosibirsk, Russia

* Author for correspondence: probatova@ibss.dvo.ru

All materials CHN; collectors: $E R=$ E.G. Rudyka, $N P=$ N.S. Probatova, $S K=$ S.G. Kazanovsky, $V L=$ V.T. Lapenko, $V N=$ V.A. Nechaev, $V S=$ V.P. Seledets.

This study was supported by grants no. 11-04-00240 (to N.S. Probatova), 11-04-01167 (to V.S. Shneyer), 12-04-01586 (to A.V. Verkhozina), from the Russian Fund for Basic Research (RFBR), and by Interdisciplinary integration project Nr. 17, from the Siberian Branch of the Russian Academy of Sciences.

\section{ADOXACEAE}

Adoxa moschatellina L., $2 n=36$; Russia, Primorskii Krai, Semeikin 12348 (VLA).

\section{ALISMATACEAE}

Alisma plantago-aquatica L., $2 n=14$; Russia, Irkutskaya Oblast', SK 12369 (VLA, IRK).

\section{ALLIACEAE}

Allium condensatum Turcz., $2 n=16$; Russia, Primorskii Krai, Prozorova 8179 (VLA).

\section{APIACEAE}

Kitagawia baicalensis (Redowsky ex Willd.) Pimenov, $2 n=22$; Russia, Republic of Buryatiya, SK 12419 (VLA, IRK).

Oenanthe javanica (Blume) DC., $2 n=22$; Russia, Primorskii Krai, Volynets \& Semeikin 12214 (VLA). $2 n=44$; Russia, Primorskii Krai, NP \& VS 7895 (VLA).

\section{ARACEAE}

Arisaema amurense Maxim., $2 n=56$; Russia, Primorskii Krai, $V N$ 12349 (VLA).

\section{ASTERACEAE}

Arctium minus (Hill.) Bernh., $2 n=36$; Russia, Primorskii Krai, $N P$ \& VS 7554 (VLA).

Artemisia scoparia Waldst. \& Kit., $2 n=16$; Russia, Republic of Buryatiya, SK 12408 (VLA, IRK).

Artemisia tanacetifolia L., $2 n=54$; Russia, Republic of Buryatiya, SK 12412 (VLA, IRK).

Glebionis segetum Fourr., $2 n=18$; Russia, Primorskii Krai, Semeikin 12217 (VLA).

Lapsana communis L., $2 n=14$; Russia, Krasnodarskii Krai, $N P$ \& VS 11510 (VLA).

Solidago dahurica Kitag., $2 n=18$; Russia, Irkutskaya Oblast', $S K$ 12372 (VLA, IRK).

Youngia tenuifolia (Willd.) Babc. \& Stebbins, $2 n=10$; Russia, Republic of Buryatiya, SK 12410 (VLA, IRK).

\section{BORAGINACEAE}

Brachybotrys paridiformis Maxim. ex Oliv., $2 n=24$; Russia, Primorskii Krai, Volynets 12139 (VLA).

\section{BRASSICACEAE}

Arabis pendula L., $2 n=16$; Russia, Primorskii Krai, VL 12355 (VLA). Erysimum cheiranthoides L., $2 n=16$; Russia, Irkutskaya Oblast', $S K$ 12385 (VLA, IRK).

Rorippa palustris (L.) Besser, $2 n=16$; Russia, Primorskii Krai, $V N$ 12424 (VLA).

\section{CARYOPHYLLACEAE}

Dianthus versicolor Fisch. ex Link, $2 n=30$; CHN. Russia, Republic of Buryatiya, SK 12362 (VLA, IRK).

Gastrolychnis brachypetala (Hornem.) Tolm. \& Kozhanch., $2 n=24$; Russia, Republic of Buryatiya, SK 12387 (VLA, IRK).

Stellaria crassifolia Ehrh., $2 n=26$; Russia, Zabaikalskii Krai, $S K$ 12375 (VLA. IRK).

Stellaria graminea L., 2n=26; Russia, Irkutskaya Oblast', SK 12409 (VLA, IRK).

Stellaria longifolia Muhl. ex Willd., $2 n=26$; Russia, Irkutskaya Oblast', SK 12416 (VLA, IRK).

\section{CONVALLARIACEAE}

Maianthemum dilatatum (Alph. Wood) A. Nelson \& J.F. Macbr., $2 n$ = 54; Russia, Primorskii Krai, ER 7998 (VLA).

\section{CONVOLVULACEAE}

Calystegia dahurica Choisy, $2 n=22$; Russia, Republic of Buryatiya, SK 12370 (VLA, IRK).

\section{CYPERACEAE}

Carex siderosticta Hance, $2 n=24$; Russia, Primorskii Krai, ER 11904 (VLA). 


\section{GERANIACEAE}

Geranium pratense L., $2 n=28$; Russia, Irkutskaya Oblast', SK 12403 (VLA, IRK).

\section{IRIDACEAE}

Iris setosa Pall. ex Link, $2 n=38$; Russia, Primorskii Krai, ER 11882 (VLA).

\section{LAMIACEAE}

Clinopodium chinense Kuntze, $2 n=20$; Russia, Primorskii Krai, $N P$ \& VS 9555 (VLA).

Dracocephalum nutans L., $2 n=10$; Russia, Irkutskaya Oblast', Prelovskaya 12396 (VLA, IRK).

Elsholtzia ciliata (Thunb.) Hyl., $2 n=16$; Russia, Republic of Altai, SK 12380 (VLA, IRK).

Lagopsis supina (Steph.) Ikonn.-Gal., $2 n=18$; Russia, Republic of Buryatiya, SK 12404 (VLA, IRK).

Leonurus glaucescens Bunge, $2 n=18$; Russia, Republic of Altai, $S K$ 12388 (VLA, IRK).

Mosla dianthera (Buch.-Ham. ex Roxb.) Maxim., $2 n=18$; Russia, Primorskii Krai, VL 12346 (VLA).

Nepeta sibirica L., 2n=18; Russia, Republic of Altai, SK 12377 (VLA, IRK).

Schizonepeta multifida (L.) Briq., $2 n=12$; Russia, Republic of Buryatiya, SK 12357 (VLA, IRK).

\section{LILIACEAE}

Lilium pilosiusculum (Freyn) Miscz., $2 n=24$; Russia, Irkutskaya Oblast', SK 12417 (VLA, IRK).

\section{LOBELIACEAE}

Lobelia sessilifolia Lamb., $2 n=28$; Russia, Primorskii Krai, VN 12422 (VLA).

\section{LYTHRACEAE}

Lythrum salicaria L., $2 n=30$; Russia, Primorskii Krai, Kapustina 12423 (VLA).

PAPAVERACEAE

Papaver olchonense Peschkova, $2 n=28$; Russia, Irkutskaya Oblast', SK 12411 (VLA, IRK)

\section{POACEAE}

Bromopsis inermis (Leyss.) Holub, $2 n=56$; Russia, Irkutskaya Oblast', SK 12407 (VLA, IRK).

Calamagrostis obtusata Trin., $2 n=28$; Russia, Republic of Altai, $S K$ 12420 (VLA, IRK)

Digitaria asiatica (Ohwi) Tzvelev, $2 n=18$; Russia, Primorskii Krai, VL 12350 (VLA), NP \& VS 7556(VLA), coll. unknown 7744 (VLA).

Digitaria ischaemum (Schreb.) Muhl., $2 n=36$; Russia, Primorskii Krai, VL 12352 (VLA)

Koeleria tokiensis Domin, $2 n=14$; Russia, Primorskii Krai, VN 8906 (VLA).

Poa alpina L., $2 n=28$; Russia, Republic of Buryatiya, SK 12344 (VLA, IRK).

Puccinellia andersonii Swallen, $2 n=42$; Russia, Primorskii Krai, Nesterova 9665 (VLA).

Puccinellia interior T.J. Sørensen, $2 n=28$; Russia, Primorskii Krai, NP \& al. 6407 (VLA).

Puccinellia paupercula Fernald \& Weath., $2 n=42$; Russia, Primorskii Krai, NP \& VS 5524 (VLA).

Puccinellia schischkinii Tzvelev, $2 n=14$; Russia, Primorskii Krai, $N P \& V S 3940$ (VLA). This specimen have been misidentified as "P. tenuissima", in Bot. Zhurn. 62(8): 1149. 1977.

Torreyochloa natans (Kom.) G.L. Church, $2 n=14$; Russia, Sakhalin, NP \& VS 5826 (VLA).

\section{POLYGALACEAE}

Polygala tenuifolia Willd., $2 n=34$; Russia, Republic of Buryatiya, SK 12398 (VLA, IRK).

PRIMULACEAE

Lysimachia volkovae Prob., $2 n=24$; Russia, Primorskii Krai, $N P$ \& VS 12418 (VLA).

\section{RANUNCULACEAE}

Aquilegia oxysepala Trautv. \& C.A. Mey., $2 n=16$; Russia, Primorskii Krai, NP \& VS 5693 (VLA).

Aquilegia turczaninovii Kamelin \& Gubanov, $2 n=16$; Russia, Zabaikal'skii Krai, SK 12360 (VLA, IRK).

\section{ROSACEAE}

Geum canadense Jacq., $2 n=42$; U.S.A., Texas, ER 9740 (VLA).

Potentilla fragarioides L., $2 n=14$; Russia, Primorskii Krai, Kapustina 10730 (VLA)

Potentilla norvegica L., $2 n=56$; Russia, Primorskii Krai, $N P \& V S$ 7824 (VLA).

Potentilla recta L., $2 n=28$; Russia, Krasnodarskii Krai, NP \& VS 12072 (VLA).

Potentilla tanacetifolia Willd. ex D.F.K. Schltdl., $2 n=28$; Russia, Republic of Buryatiya, SK 12338 (VLA, IRK).

\section{SCROPHULARIACEAE}

Veronica linariifolia Pall. ex Link, $2 n=34$; Russia, Republic of Buryatiya, SK 12374 (VLA, IRK).

\section{SOLANACEAE}

Solanum nigrum L., $2 n=72$; Russia, Primorskii Krai, VL 12339 (VLA).

\section{VALERIANACEAE}

Patrinia rupestris (Pall.) Juss., $2 n=22$; Russia, Republic of Buryatiya, SK 12371 (VLA, IRK).

\section{Nina S. Probatova, ${ }^{1 *}$ Alla V. Verkhozina, ${ }^{2}$ Elvira G. Rudyka, ${ }^{1}$} \& Denis A. Krivenko ${ }^{2}$

1 Institute of Biology \& Soil Science, Far East Branch of the Russian Academy of Sciences, 159 Stoletya Prospect, 690022, Vladivostok, Russia

2 Siberian Institute of Plant Physiology \& Biochemistry, Siberian Branch of the Russian Academy of Sciences, 132 Lermontov Str. 664033, Irkutsk, Russia

* Author for correspondence:probatova@ibss.dvo.ru

\section{All materials CHN.}

This study was supported by grants no. 11-04-00240 (to N.S Probatova), 12-04-01586 (to A.V. Verkhozina), 12-04-31151 (to D.A. Krivenko) from the Russian Fund for Basic Research (RFBR), and by Interdisciplinary integration project Nr. 17, from the Siberian Branch of the Russian Academy of Sciences.

\section{ALLIACEAE}

Allium ramosum L., $2 n=32$; Russia, Amurskaya Oblast', Kudrin 12330 (VLA).

\section{ASTERACEAE}

Erigeron elongatus Ledeb., $2 n=18$; Russia, Irkutskaya Oblast', Kazanovsky \& Verkhozina 12373 (VLA, IRK).

Galatella dahurica DC., $2 n=18$; Russia, Republic of Sakha-Yakutia, Krivenko 12414 (VLA, IRK). 
Lactuca serriola L., $2 n=18$; Russia, Amurskaya Oblast', Kudrin 12323 (VLA).

Matricaria perforata Mérat, $2 n=36$; Russia, Irkutskaya Oblast', Verkhozina \& Tolstonogova 12384 (VLA, IRK).

Pterocypsela indica (L.) C. Shih, $2 n=18$; Russia, Amurskaya Oblast', Kudrin 12324 (VLA).

Tanacetum vulgare L., $2 n=18$; Russia, Republic of Sakha-Yakutia, Krivenko 12415 (VLA, IRK).

\section{BORAGINACEAE}

Echium vulgare L., $2 n=16$; Russia, Irkutskaya Oblast', Verkhozina 12359 (VLA, IRK).

Lappula lenensis Popov ex Ovczinnikova, $2 n=24$; Russia, Republic of Sakha-Yakutia, Verkhozina 12395 (VLA, IRK).

\section{BRASSICACEAE}

Bunias orientalis L., $2 n=14$; Russia, Irkutskaya Oblast', Verkhozina \& al. 12391 (VLA, IRK).

Descurainia sophia (L.) Webb ex Prantl, $2 n=14$; Russia, Irkutskaya Oblast', Verkhozina \& Kazanovsky 12382 (VLA, IRK).

Lepidium latifolium L., $2 n=24$; Russia, Irkutskaya Oblast', Verkhozina \& al. 12390 (VLA, IRK).

\section{CARYOPHYLLACEAE}

Lychnis chalcedonica L., $2 n=24$; Russia, Irkutskaya Oblast', Kazanovsky \& Verkhozina 12402 (VLA, IRK).

\section{FABACEAE}

Astragalus frigidus (L.) A. Gray, $2 n=16+0-2 \mathrm{~B}$; Russia, Republic of Buryatiya, Krivenko 29593 (IRK).

Astragalus mongholicus Bunge, $2 n=16$; Russia, Irkutskaya Oblast', Prelovskaya \& Bondarevich 28970 (IRK).

Astragalus sericeocanus Gontsch., $2 n=16+0-1 \mathrm{~B}$, Russia, Republic of Buryatiya, Zhmud' 23490 (IRK).

\section{LAMIACEAE}

Dracocephalum thymiflorum L., $2 n=14$; Russia, Irkutskaya Oblast', Verkhozina \& al. 12386 (VLA, IRK).

Leonurus villosus Desf., $2 n=18$; Russia, Irkutskaya Oblast', Verkhozina \& Kaverzina 12379 (VLA, IRK)

LYTHRACEAE

Lythrum salicaria L., $2 n=30$; Russia, Irkutskaya Oblast', Verkhozina \& al. 12381 (VLA, IRK).

PAPAVERACEAE

Papaver nudicaule L., $2 n=28$; Russia, Republic of Buryatiya, Verkhozina 12392 (VLA, IRK).

PLANTAGINACEAE

Plantago major L., $2 n=12$; Russia, Irkutskaya Oblast', Verkhozina 12421 (VLA, IRK).

POACEAE

Agrostis clavata Trin., 2n=42; Russia, Amurskaya Oblast', Parilova 12311 (VLA).
Digitaria asiatica Tzvelev, $2 n=18$; Russia, Khabarovskii Krai, Probatova \& Seledets 10484 (VLA).

Setaria glareosa Petrov, $2 n=18$; Russia, Irkutskaya Oblast', Kazanovsky \& Verkhozina 12376 (VLA, IRK).

Setaria pumila (Poir.) Roem. \& Schult., $2 n=18$; Russia, Amurskaya Oblast', Yashenkova 6302 (VLA). $2 n=36$; Russia, Volgogradskaya Oblast', Yurtseva 10496 (VLA).

\section{PORTULACACEAE}

Portulaca oleracea L., $2 n=36$; Russia, Amurskaya Oblast', Kudrin 12319 (VLA).

\section{ROSACEAE}

Comarum palustre L., $2 n=28$; Russia, Irkutskaya Oblast', Verkhozina \& Kazanovsky 12401 (VLA, IRK).

Potentilla longifolia Willd. ex D.F.K. Schltdl., $2 n=14$; Russia, Republic of Sakha-Yakutia, Krivenko 12337 (VLA, IRK).

Potentilla norvegica L., $2 n=56$; Russia, Irkutskaya Oblast', Verkhozina 12383 (VLA, IRK).

Potentilla paradoxa Nutt. ex Torr. \& Gray, $2 n=28$; Russia, Republic of Sakha-Yakutia, Verkhozina 12413 (VLA, IRK); Russia, Republic of Buryatiya, Verkhozina \& Anossova 12340 (VLA, IRK).

\section{María Santos-Vicente, * Enrique Rico \& M. Montserrat Martínez-Ortega}

Departamento de Botánica, Universidad de Salamanca, 37007

Salamanca, Spain

* Author for correspondence: m.santos@usal.es

All materials CHN; collectors: $A A=\mathrm{A}$. Abad, $A G=\mathrm{A}$. Guillén, $A H=\mathrm{A}$. Herrero, $A Q=\mathrm{A}$. Quintanar, $C M=\mathrm{C}$. Molina, $C N=$ C. Navarro, $J A=$ J.A. Sánchez-Agudo, $L D=\mathrm{L}$. Delgado-Sánchez, $M O$ $=$ M.M. Martínez-Ortega, $M S=$ M. Santos-Vicente, $P B=$ P. Bariego, $S A=\mathrm{S}$. Andrés-Sánchez, $X G=\mathrm{X}$. Giráldez; vouchers in SALA.

This study was supported by the Spanish Dirección General de Investigación (Ministerio de Ciencia y Tecnología and Ministerio de Educación y Ciencia) through the projects Flora iberica VI (REN2002-04634-C05-02; grant ref. BES-2003-2213 to M. Santos Vicente) and Flora iberica VII (CGL2005-05471-C04-03) and by the Junta de Castilla y León through project SA142A08. We greatly acknowledge this support.

\section{ASTERACEAE}

Inula helenioides DC., $2 n=32$; Spain, $A A, S A, M O \& M S$ 758. $2 n=$ 40; Spain, $L D \& M S$ 907; Spain, $L D, C M \& M S 953 b$.

Inula langeana Beck, $2 n=32$; Spain, $S A$, $J A \& M S$ 412. $2 n=40$; Spain, $P B \& M S 583.2 n=48$; Spain, $L D \& M S$ 952, AA, $S A, M O \&$ MS 755, LD \& MS 946.

Inula maletii Maire, 2n=32; Morocco, AH 3347, AQ 2043, AQ 2153. Inula montana L., $2 n=16$; Spain, $L D \&$ \&S 882; Morocco, AH 3215. Inula oculus-christi L., $n=16$; Bulgaria, CN 4769.

Inula salicina L., $2 n=16$; Spain, $X G, A G \& M S 404, L D \& M S 572$. 


\section{IOPB COLUMN}

\section{Edited by Karol Marhold \& Ilse Breitwieser}

\section{IAPT/IOPB chromosome data 16}

\section{Edited by Karol Marhold}

\section{Laura Chalup ${ }^{1 *} \&$ Guillermo Seijo ${ }^{1,2}$}

1 Instituto de Botánica del Nordeste (UNNE-CONICET), Facultad de Ciencias Agrarias, Sgto. Cabral 2131, 3400 Corrientes, Argentina

2 Facultad de Ciencias Exactas y Naturales y Agrimensura, Universidad Nacional del Nordeste, Avenida Libertad 5500 3400 Corrientes, Argentina

* Author for correspondence: lchalup@agr.unne.edu.ar

This work was supported by Agencia Nacional de Promoción Científica y Tecnológica, Argentina PICTO-UNNE 090. Laura Chalup is a fellow of Consejo Nacional de Investigaciones Científicas y Técnicas (CONICET), Argentina.

* First chromosome count for the species or variety.

$\nabla$ First chromosome count for a given country.

\section{FABACEAE}

Lathyrus crassipes Hook. \& Arn.

$2 n=14$, CHN. Argentina, Corrientes, Empedrado, El Sombrero Experimental Station of National Institute of Agronomic Technology

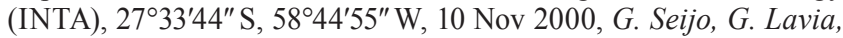
H. Roig \& H. Bogado 2349 (CTES, G, U).

Lathyrus latifolius $\mathrm{L}$.

$2 n=14$, CHN. Argentina, Córdoba, Punilla, Capilla del Monte, 3051'17"S, 64³1'33" W, 11 Nov 2005, V. Solis Neffa 1998 (CTES) [Fig. 1A].

$n=7$, CHN. Argentina, Río Negro, Bariloche, Puerto Pañuelo, $41^{\circ} 03^{\prime} 13^{\prime \prime}$ S, 71³1'58" W, 7 Feb 1998, G. Seijo 1170 (CTES, LIL, SPF).

Lathyrus macrostachys Vogel

$n=7$, CHN. Argentina, Misiones, Posadas, Zaimán river, $27^{\circ} 26^{\prime} 48^{\prime \prime}$ S, 55 54'12.1" W, 3 Dec 2008, L. Chalup, G. Seijo, V. Solis Neffa \& A. González 1 (CTES).

Lathyrus magellanicus var. glaucescens Speg.

$* 2 n=14$, CHN. Argentina, Chubut, Languiñeo, Corcovado river, 43 $53^{\prime} 53^{\prime \prime} \mathrm{S}, 7^{\circ} 25^{\prime} 30^{\prime \prime} \mathrm{W}, 2$ Feb 2009, L. Chalup, W. Medina, C. Mackluf \& E. Meza Torres 19 (CTES, MO, NY) [Fig. 1C].

Lathyrus magellanicus Lam. var. magellanicus

$2 n=14$, CHN. Argentina, Río Negro, Bariloche, Gutiérrez lake, 41 ${ }^{\circ} 13^{\prime} 25^{\prime \prime}$ S, 7124'43" W, 17 Jan 2000, G. Seijo 2307 (CTES, K, NY, SI) [Fig. 1B].

Lathyrus magellanicus var. tucumanensis Burkart

$n=7$, CHN. Argentina, Jujuy, Tiraxi, 2000'19" S, 64²1'24" W, 19 Sep 1997, G. Seijo \& M. Dematteis 1109 (CEN, CTES, LPB, MBM, NY).
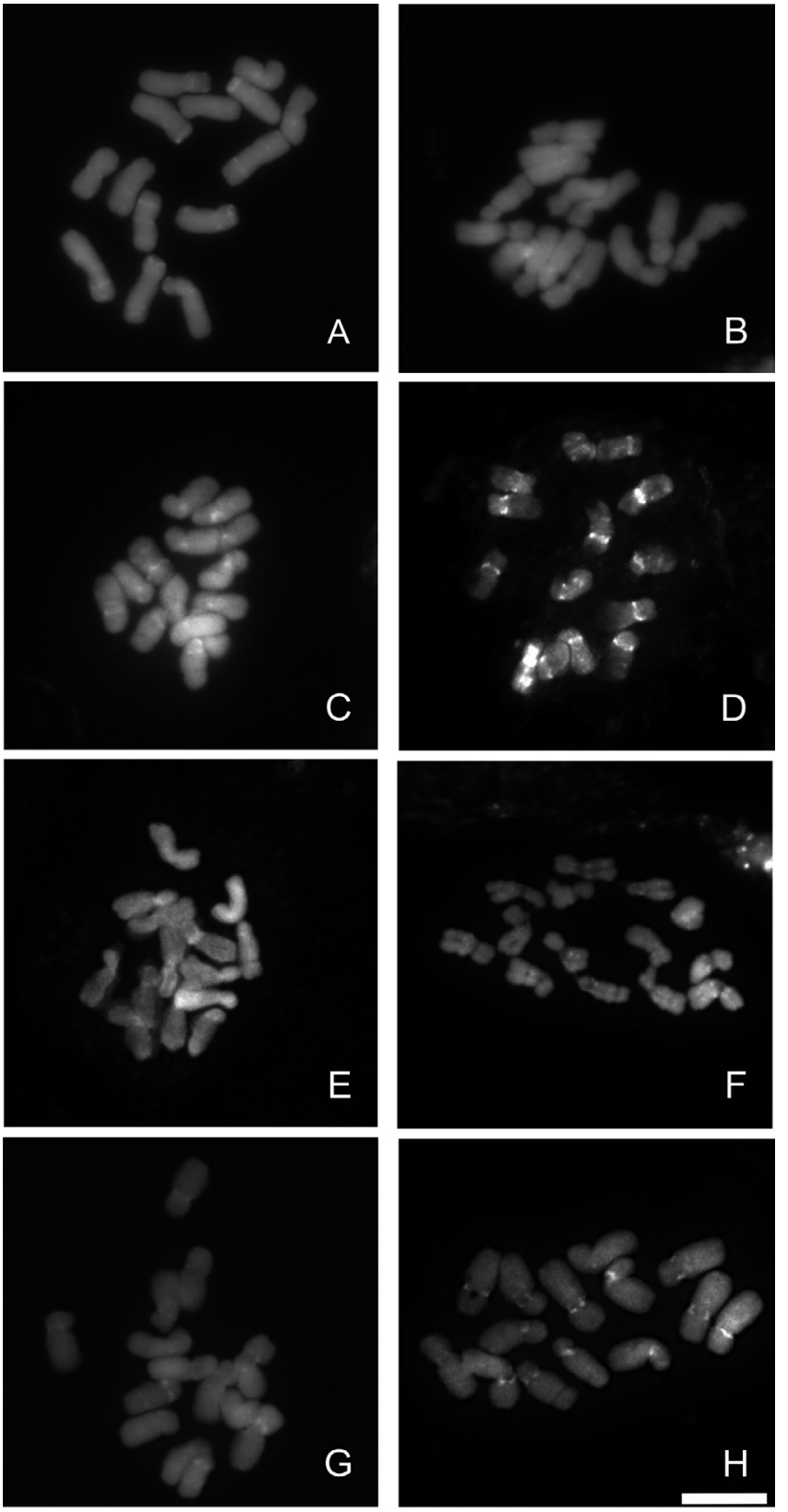

Fig. 1. Mitotic metaphases of Lathyrus stained with DAPI. A, L. latifolius (V. Solís Neffa 1998), $2 n=14 ; \mathbf{B}$, L. magellanicus var. magellanicus (G. Seijo 2307), $2 n=14 ; \mathbf{C}$, L. magellanicus var. glaucescens (L. Chalup \& al. 19), $2 n=14$; D, L. multiceps (G. Seijo 1607), $2 n=14$; $\mathbf{E}, L$. nitens (G. Seijo \& V. Solís Neffa 3966), $2 n=14 ; \mathbf{F}, L$. paranensis (G. Seijo \& V. Solís Neffa 3954), $2 n=14 ; \mathbf{G}$, L. pubescens $(G$. Seijo \& V. Solís Neffa 3972), $2 n=14 ; \mathbf{H}$, L. pubescens (G. Seijo 2332), $2 n=$ 14. - Scale bar $=10 \mu \mathrm{m}$.

All materials for the chromosome column should be submitted electronically to: Karol Marhold, karol.marhold@savba.sk (Institute of Botany, Slovak Academy of Sciences, SK-845 23 Bratislava, Slovakia, and Department of Botany, Charles University, CZ 128-01 Prague, Czech Republic). The full version of this contribution is available in the online edition of TAXON appended to this article. The following citation format is recommended: Baltisberger, M. \& Voelger, M. 2006. Sternbergia sicula. In: Marhold, K. (ed.), IAPT/IOPB chromosome data 1. Taxon 55: 444, E2. 
Lathyrus multiceps Clos

$2 n=14, \mathrm{CHN}$. Argentina, Neuquén, Los Lagos, Correntoso river, 4044'13" S, 7140'15" W, 28 Jan 1999, G. Seijo 1607 (CTES, F, TEX) [Fig. 1D].

$n=7$, CHN. Argentina, Neuquén, Lacar, Hermoso lake, 40²1'40" S, 712ㅇ'36" W, 10 Feb 1998, G. Seijo 1194 (CTES, K, MICH); Argentina, Neuquén, Aluminé, Villa Pehuenia, 38 $53^{\prime} 37^{\prime \prime}$ S, $71^{\circ} 11^{\prime} 09^{\prime \prime}$ W, 3 Feb 1998, G. Seijo 1653 (ALCB, CTES, G, HUEFS, MBM).

Lathyrus nitens Vogel

$* 2 n=14$, CHN. Uruguay, Tacuarembó, road to Gruta de los Cuervos, $31^{\circ} 37^{\prime} 12^{\prime \prime} \mathrm{S}, 56^{\circ} 02^{\prime} 30^{\prime \prime} \mathrm{W}, 17$ Nov 2007, G. Seijo \& V. Solís Neffa 3966 (CTES, UTEP) [Fig. 1E].

Lathyrus paranensis Burkart

$\boldsymbol{\nabla} 2 n=14$, CHN. Uruguay, Rivera, route 29, Cañada de Santa

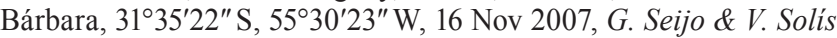
Neffa 3954 (ASU, BGE, CTES, FUEL, I, MBM) [Fig. 1F]; Uruguay,

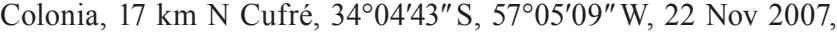
G. Seijo \& V. Solís Neffa 3984 (CTES).

Lathyrus pubescens Hook. \& Arn.

$\boldsymbol{\nabla} 2 n=14$, CHN. Uruguay, Rivera, Bajada de Pena, $31^{\circ} 08^{\prime} 35^{\prime \prime} \mathrm{S}$, 55 54'59" W, 15 Nov 2007, G. Seijo \& V. Solís Neffa 3941 (CTES, FUEL, IAC, ICN, MBM, MO); Uruguay, San José, Balneário Kiyú, 31 41'36" S, 5644'55" W, 21 Nov 2007, G. Seijo \& V. Solís Neffa 3972 (CEN, CTES) [Fig. 1G]; Argentina, Buenos Aires, Tornquist, route $72,10 \mathrm{~km} \mathrm{~S}$ from Saldungaray to Paso de Piedras dum, $38^{\circ} 05^{\prime} 29^{\prime \prime} \mathrm{S}$, $62^{\circ} 16^{\prime} 13^{\prime \prime} \mathrm{W}, 20$ Jan 2000, G. Seijo 2332 (CTES) [Fig. 1H].

$n=7$, CHN. Argentina, Tucumán, road from Anta Muerta to Villa Noguéz, 2651'32" S, 65²1'09" W, 18 Sep 1997, G. Seijo \& M. Dematteis 1086 (CTES, CUVC, HUEFS, MBM, MICH, NY); Argentina, San Luis, Chacabuco, Villa Larca, Chorro de San Ignacio, 32³8'32" S, 6456'47" W, 13 Dec 1999, G. Seijo 2059 (CTES); Bolivia, Santa Cruz, Caballero, $8 \mathrm{~km} \mathrm{~W}$ from Comarapa to Cocha-

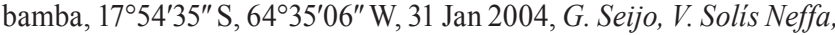
A. Schinini \& R. Almada 3082 (CTES).

\section{Neli H. Grozeva}

Department of Biology and Aquaculture, Agricultural Faculty, Trakia University,6000 Stara Zagora, Bulgaria; grozeva@uni-sz.bg

This study was supported by the Project Scientific Research Fund of Trakia University, Agriculture faculty.

\section{CHENOPODIACEAE}

Atriplex oblongifolia Waldst. \& Kit.

$2 n=36$, CHN. Bulgaria, the Black Sea Coast, Nessebar old town, sandy terrains in the east end of the beach with Chenopodium vulvaria L. and C. polyspermum L., 42 $39^{\prime} \mathrm{N}, 27^{\circ} 44^{\prime} \mathrm{E}, 0$ m, 22 Sep 2010, N.H. Grozeva 4058 (SOM) [Fig. 2A].

Atriplex tatarica $\mathrm{L}$.

$2 n=18$, CHN. Bulgaria, the Black Sea Coast, Varnensko ezero lake, periodically flooded coastal alluvial saline sands, along coast-

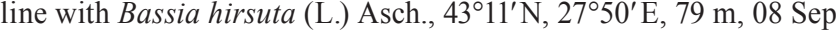
2011, N.H. Grozeva 4059 (SOM) [Fig. 2B].

\section{Bassia hirsuta (L.) Asch.}

$2 n=18$, CHN. Bulgaria, the Black Sea Coast, Varnensko ezero lake, periodically flooded coastal alluvial sands, along coastline with Atriplex tatarica, $43^{\circ} 11^{\prime} \mathrm{N}, 27^{\circ} 50^{\prime} \mathrm{E}, 79 \mathrm{~m}, 08 \mathrm{Sep} 2011$, N.H. Grozeva 4060 (SOM) [Fig. 2C].
Bassia laniflora (S.G. Gmel.) A.J. Scott

$2 n=18$, CHN. Bulgaria, the Black Sea Coast, Ahtopol town, near the coastline south of the town with Corispermum nitidum Kit. ex Schult., Salsola tragus L., Chenopodium pratericola Rydb. etc., $42^{\circ} 05^{\prime} 40^{\prime \prime} \mathrm{N} 27^{\circ} 56^{\prime} 60^{\prime \prime}$ E, 6 m, 09 Sep 2011, N.H. Grozeva 4062 (SOM) [Fig. 2D].

This is the first chromosome count for the species from Bulgarian populations.

Chenopodium album $\mathrm{L}$.

$2 n=54$, CHN. Bulgaria, Sredna Gora Mt., Gorno Novo selo village, ruderal places at the western end of the village near the road for the hut Kavakliika, 42 $27^{\prime} \mathrm{N}, 25^{\circ} 14^{\prime} \mathrm{E}, 597$ m, 17 Sep 2010 , N.H. Grozeva 4061 (SOM) [Fig. 2E].

Chenopodium chenopodioides (L.) Aellen

$2 n=18$, CHN. Bulgaria, the Black Sea Coast, Obzor town, near the coastline north of the town with Eryngium maritimum L. and Crambe maritima L., 42 $49^{\prime} 43^{\prime \prime} \mathrm{N}, 2^{\circ} 52^{\prime} 55^{\prime \prime} \mathrm{E}, 1 \mathrm{~m}, 22$ Sep 2011, N.H. Grozeva 4063 (SOM) [Fig. 2F].

Chenopodium polyspermum $\mathrm{L}$.

$2 n=18$, CHN. Bulgaria, Thracian Lowland, Asenovgrad town, ruderal places on the territory of the health centre Euromed with Polygonum aviculare L., $42^{\circ} 01^{\prime} \mathrm{N}, 2^{\circ} 52^{\prime} \mathrm{E}, 104$ m, 18 Sep 2010, N.H. Grozeva 4064 (SOM) [Fig. 2G].

Chenopodium rubrum $\mathrm{L}$.

$2 n=18$, CHN. Bulgaria, Thracian Lowland, Stara Zagora town, around the trunk of Tilia tomentosa in the Trite chuchura neighborhood, $42^{\circ} 25^{\prime} \mathrm{N}, 25^{\circ} 38^{\prime} \mathrm{E}, 195$ m, 25 Sep 2010, N.H. Grozeva 4067 (SOM) [Fig. 2H].

Chenopodium strictum Roth

$2 n=36$, CHN. Bulgaria, Thracian Lowland, Purvomay town, degraded land between the houses along Viktor Yugo street in Debar neighborhood, $42^{\circ} 06^{\prime} \mathrm{N}, 25^{\circ} 13^{\prime} \mathrm{E}, 134 \mathrm{~m}, 28 \mathrm{Sep} 2010$, N.H. Grozeva 4065 (SOM) [Fig. 2I].

Chenopodium urbicum $\mathrm{L}$.

$2 n=18$, CHN. Bulgaria, Thracian Lowland, Tselina village, ruderal places east of the gas station along the road to Velikan village with Chenopodium album, $42^{\circ} 07^{\prime} \mathrm{N}, 25^{\circ} 27^{\prime} \mathrm{E}, 193 \mathrm{~m}, 27$ Sep 2010, N.H. Grozeva 4066 (SOM) [Fig. 2J].

Corispermum nitidum Kit. ex Schult.

$2 n=18$, CHN. Bulgaria, the Black Sea Coast, Ahtopol town, near the coastline south of the town with Corispermum nitidum, Bassia laniflora, Salsola tragus, Chenopodium pratericola, etc., $42^{\circ} 05^{\prime} \mathrm{N}$ $27^{\circ} 56^{\prime}$ E, 6 m, 09 Sep 2011, N.H. Grozeva 4068 (SOM) [Fig. 2K].

Dysphania pumilio (R. Br.) Mosyakin \& Clemants

$2 n=18$, CHN. Bulgaria, Rhodope Mts., Kurdzhali town, ruderal places between buildings in Studen kladenec neighborhood with $D y s-$ phania botrys (L.) Mosyakin \& Clemants, $41^{\circ} 39^{\prime} \mathrm{N}, 25^{\circ} 38^{\prime} \mathrm{E}, 275 \mathrm{~m}$, 10 Sep 2011, N.H. Grozeva 4069 (SOM) [Fig. 2L].

Salicornia europaea L.

$2 n=18$, CHN. Bulgaria, the Black Sea Coast, Pomoriysko lake, in saline areas around the lake near Salt museum with Bassia hirsuta and Suaeda maritima (L.) Dumort., 42 $35^{\prime} \mathrm{N}, 27^{\circ} 37^{\prime} \mathrm{E}, 10 \mathrm{~m}$, 08 Sep 2011, N.H. Grozeva 4070 (SOM) [Fig. 2M].

This is the first chromosome count for the species from Bulgarian populations. 

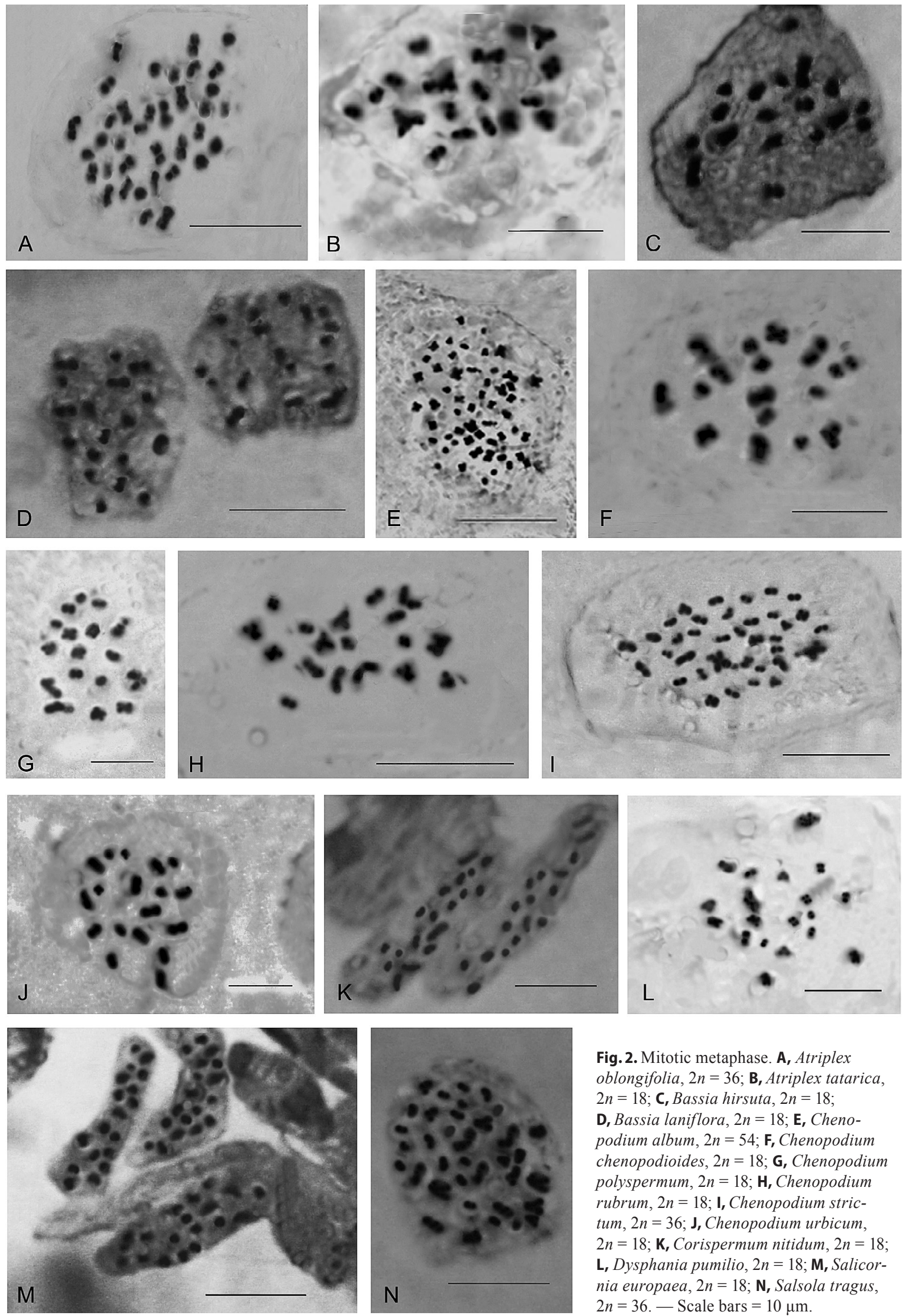

Fig. 2. Mitotic metaphase. A, Atriplex oblongifolia, $2 n=36$; B, Atriplex tatarica, $2 n=18 ; \mathbf{C}$, Bassia hirsuta, $2 n=18$; D, Bassia laniflora, $2 n=18 ; \mathbf{E}$, Chenopodium album, $2 n=54 ; \mathbf{F}$, Chenopodium chenopodioides, $2 n=18 ; \mathbf{G}$, Chenopodium polyspermum, $2 n=18 ; \mathbf{H}$, Chenopodium rubrum, $2 n=18$; I, Chenopodium strictum, $2 n=36$; J, Chenopodium urbicum, $2 n=18 ; \mathbf{K}$, Corispermum nitidum, $2 n=18$; L, Dysphania pumilio, $2 n=18$; M, Salicornia europaea, $2 n=18 ; \mathbf{N}$, Salsola tragus, $2 n=36$. - Scale bars $=10 \mu \mathrm{m}$. 
Salsola tragus $\mathrm{L}$.

$2 n=36$, CHN. Bulgaria, the Black Sea Coast, Ahtopol town, near the coastline south of the town with Corispermum nitidum, Bassia laniflora, Chenopodium pratericola, etc., 09 Sep $2011,42^{\circ} 05^{\prime} 40^{\prime \prime} \mathrm{N}$ $27^{\circ} 56^{\prime} 60^{\prime \prime}$ E, 6 m, N.H. Grozeva 4071 (SOM) [Fig. 2N].

This is the first chromosome count for the species from Bulgarian populations.

Denis A. Krivenko, ${ }^{*}$ Violetta V. Kotseruba, ${ }^{2}$ Sergey G. Kazanovsky, ${ }^{1}$ Alla V. Verkhozina ${ }^{1} \&$ Olga D. Chernova ${ }^{3}$

1 Institute of Plant Physiology \& Biochemistry Siberian Branch of the Russian Academy of Sciences, 132 Lermontov Str., 664033, Irkutsk, Russia

2 Komarov Botanical Institute of the Russian Academy of Sciences, 2 Prof. Popov Str., 197376, St.-Petersburg, Russia

3 Zabaikalsky State Humanitarian Pedagogical University named after N. Chernishevsky, 129 Babushkina Str., 672007, Chita, Russia

* Author for correspondence: krivenko.irk@gmail.com

* First chromosome count for the genus.

** First chromosome count for the species.

*** New chromosome number (cytotype) for the species.

This study was supported by grants no. 11-04-00240-a, 11-0401167-a, 12-04-01586-a, 12-04-31151 mol-a, 13-04-00351-a, 13-0401458-a, 13-04-01468-a from the Russian Fund for Basic Research (RFBR) and by Interdisciplinary integration project Nr. 17, from the Siberian Branch of the Russian Academy of Sciences.

\section{ACORACEAE}

Acorus calamus L.

$2 n=24$, CHN. Russia, Republic of Buryatiya, Pribaikal'skii Raion, vicinity of Gremyachinsk village, $411 \mathrm{~m}, 52^{\circ} 47^{\prime} 46^{\prime \prime} \mathrm{N}$, $107^{\circ} 58^{\prime} 44^{\prime \prime} \mathrm{E}$, on the marshy shore of the lake on the shore of Baikal Lake, 22 Jul 2011, A.V. Verkhozina 19864 (IRK).

\section{ADOXACEAE}

Adoxa moschatellina $\mathrm{L}$.

$2 n=36$, CHN. Russia, Republic of Altai, Ongudaiskii Raion, left bank of the Aigulak River, near the mouth, $1149 \mathrm{~m}, 50^{\circ} 12^{\prime} 42^{\prime \prime} \mathrm{N}$, $087^{\circ} 14^{\prime} 45^{\prime \prime} \mathrm{E}$, on the rocks covered with moss, $28 \mathrm{Jul} 2012$, D.A. Krivenko 29573 (IRK).

\section{APIACEAE}

Conium maculatum $\mathrm{L}$.

$2 n=22$, CHN. Russia, Novosibirskaya Oblast', Novosibirsk city, Akademgorodok, vicinity of Central Siberian Botanical Garden, Siberian Branch of the Russian Academy of Sciences, right bank of the Zyryanka River, $145 \mathrm{~m}, 54^{\circ} 49^{\prime} 25^{\prime \prime} \mathrm{N}, 083^{\circ} 06^{\prime} 08^{\prime \prime} \mathrm{E}$, vegetation ruderal on the road, 4 Sep 2012, S.G. Kazanovsky 26612 (IRK).

\section{ASCLEPIADACEAE}

Vincetoxicum sibiricum (L.) Decne.

$2 n=22$, CNH. Russia, Zabaikal'skii Krai, Krasnokamenskii Raion, Krasnokamensk town, mining dumps, Urtui dump, steppe, 20 Aug 2009, Ye.A. Bondarevich 29562 (IRK).

\section{ASTERACEAE}

Artemisia halodendron Turcz. ex Besser

$2 n=36$, CNH. Mongolia, Dornod Aimak, Khalkin-Gol somon, steeply sloping plain, $10 \mathrm{~km}$ E of the Buyr-Nur Lake, deep sandy gully, aeolian sands, 15 Aug 2008, A.A. Korobkov 09-92 (LE).
Artemisia insulana Krasch.

$2 n=18$, CNH. Russia, Kamchatskii Krai, Aleutskii Raion, Pacific Ocean, Bering Sea, Komandorskie Islands, Beringa Island, slope of the ridge, shrub tundra, 24 Aug 1999, N.V. Sinel'nikova 99203 (LE).

\section{Artemisia tanacetifolia $\mathrm{L}$.}

$2 n=54$, CNH. Russia, Altaiskii Krai, vicinity of Barnaul city, experimental plot South Siberian Botanical Garden, 19 Sep 1999, A.A. Korobkov 99-196 (LE).

Artemisia vulgaris L.

$2 n=16$, CNH. Russia, Kemerovskaya Oblast', Tashtagol'skii Raion, vicinity of Kaz village, grass-motley group at the highway, 11 Sep 2000, I.M. Krasnoborov 00-111 (NS).

Cirsium esculentum (Siev.) C.A. Mey.

$2 n=34$, CHN. Russia, Irkutskaya Oblast', Ol'khonskii Raion, $2 \mathrm{~km}$ NE from the Zama village (through rocky spit), flow between Baikal Lake and Zama Lake, $454 \mathrm{~m}, 53^{\circ} 28^{\prime} 18^{\prime \prime} \mathrm{N}, 107^{\circ} 32^{\prime} 21^{\prime \prime} \mathrm{E}$, marshy shore of Zama Lake, 24 Aug 2011, A.V. Verkhozina 19730 (IRK).

Neopallasia pectinata (Pall.) Poljakov

$2 n=18$, CNH. Russia, Republic of Buryatiya, Selenginskii Raion, Borgoiskii Mts., north slope of the ridge to the Selenduma village, broken sand-gravel sites along of the edge road, 18 Sep 2005, A.A. Korobkov 06-211 (LE).

Petasites frigidus (L.) Fr.

$2 n=60$, CHN. Russia, Republic of Sakha (Yakutia), Mirninskii Raion, right bank of the Byuryuelyakh River (right bank of the Ulakhan-Botuobuya River), $6.5 \mathrm{~km}$ upstream from the mouth river, $316 \mathrm{~m}, 61^{\circ} 12^{\prime} 28^{\prime \prime} \mathrm{N}, 112^{\circ} 08^{\prime} 11^{\prime \prime} \mathrm{E}$, spruce-larch blueberry lichengreenmoss forest, 1 Sep 2012, D.A. Krivenko 27259 (IRK).

Senecio vulgaris $\mathrm{L}$.

$2 n=40$, CHN. Russia, Sverdlovskaya Oblast', Ekaterinburg city, territory of Institute of Plant \& Animal Ecology, Ural Branch of the Russian Academy of Sciences, old building, 56 $49^{\prime} 20^{\prime \prime} \mathrm{N}$, $060^{\circ} 19^{\prime} 38^{\prime \prime} \mathrm{E}$, ruderal community along the asphalt walkway, 7 Sep 2011, O.S. Dymshakova 21110 (IRK).

Sonchus asper (L.) Hill

$2 n=18$, CHN. Russia, Sverdlovskaya Oblast', Ekaterinburg city, territory of Institute of Plant \& Animal Ecology, Ural Branch of the Russian Academy of Sciences, old building, 56 $49^{\prime} 20^{\prime \prime} \mathrm{N}$, $060^{\circ} 19^{\prime} 38^{\prime \prime}$ E, ruderal community along the asphalt walkway, 7 Sep 2011, O.S. Dymshakova 21105 (IRK).

Taraxacum officinale F.H. Wigg.

$2 n=24$, CHN. Russia, Sverdlovskaya Oblast', Ekaterinburg city, territory of Institute of Plant \& Animal Ecology, Ural Branch of the Russian Academy of Sciences, old building, 56 $49^{\prime} 20^{\prime \prime} \mathrm{N}$, $060^{\circ} 19^{\prime} 38^{\prime \prime} \mathrm{E}$, ruderal community along the asphalt walkway, $7 \mathrm{Sep}$ 2011, O.S. Dymshakova 29603 (IRK).

Tragopogon orientalis L.

$2 n=12$, CHN. Russia, Altaiskii Krai, Volchikhinskii Raion, vicinity of Volchikha village, Volchikhinskiye barrows, among arable, $277 \mathrm{~m}, 52^{\circ} 12^{\prime} 18^{\prime \prime} \mathrm{N}, 080^{\circ} 30^{\prime} 16^{\prime \prime} \mathrm{E}$, lode, 17 Jul 2012, D.A. Krivenko 29637 (IRK).

**Tragopogon trachycarpus S.A. Nikitin

$2 n=12$, CHN. Russia, Republic of Buryatiya, Pribaikal'skii Raion, $4 \mathrm{~km} \mathrm{~S}$ of the Turuntaevo village, $480 \mathrm{~m}, 52^{\circ} 11^{\prime} 32^{\prime \prime} \mathrm{N}$, 
$107^{\circ} 35^{\prime} 25^{\prime \prime} \mathrm{E}$, escarpment of the road, 22 Jul 2011, D.A. Krivenko 28217 (IRK) [Fig. 3H]

Tridactylina kirilowii (Turcz.) Sch. Bip.

$2 n=18$, CHN. Russia, Irkutskaya Oblast', Slyudyanskii Raion, lower reaches of Slyudyanka River, 51 $29^{\prime} 57^{\prime \prime} \mathrm{N}, 104^{\circ} 09^{\prime} 33^{\prime \prime}$ E, coastal pebbles, 28 Aug 2010, Yu.N. Pochinchik 24539 (IRK) [Fig. 3I].

\section{BORAGINACEAE}

* Craniospermum subvillosum Lehm.

$2 n=24, \mathrm{CNH}$. Russia, Republic of Buryatiya, Pribaikal'skii Raion, Baikal Lake, vicinity of Gremyachinsk village, $411 \mathrm{~m}, 52^{\circ} 47^{\prime} 46^{\prime \prime} \mathrm{N}$, $107^{\circ} 58^{\prime} 44^{\prime \prime} \mathrm{E}$, sandy shore, 22 Jul 2011, D.A. Krivenko 23123 (IRK).

** Lappula tuvinica Ovczinnikova

$2 n=24$, CHN. Russia, Altaiskii Krai, Tret'yakovskii Raion, Staroaleiskoe village, $301 \mathrm{~m}, 51^{\circ} 00^{\prime} 25^{\prime \prime} \mathrm{N}, 082^{\circ} 00^{\prime} 31^{\prime \prime} \mathrm{E}$, roadside, 19 Jul 2012, D.A. Krivenko 29604 (IRK).

\section{BRASSICACEAE}

Alyssum tortuosum Waldst. \& Kit. ex Willd.

$2 n=16$, CHN. Russia, Altaiskii Krai, Mikhailovskii Raion, near Malinovoye Lake, $163 \mathrm{~m}, 51^{\circ} 39^{\prime} 50^{\prime \prime} \mathrm{N}, 079^{\circ} 49^{\prime} 59^{\prime \prime} \mathrm{E}$, steppe, $17 \mathrm{Jul}$ 2012, D.A. Krivenko 29633 (IRK).

Capsella bursa-pastoris (L.) Medik.

$2 n=32$, CHN. Russia, Sverdlovskaya Oblast', Ekaterinburg city, territory of Institute of Plant \& Animal Ecology, Ural Branch of the Russian Academy of Sciences, old building, 56 49'20" N, $060^{\circ} 19^{\prime} 38^{\prime \prime} \mathrm{E}$, ruderal community along the asphalt walkway, $7 \mathrm{Sep}$ 2011, O.S. Dymshakova 21108 (IRK).

Lepidium ruderale $\mathrm{L}$.

$2 n=16$, CHN. Russia, Irkutskaya Oblast', Irkutskii Raion, vicinity of Maloe Goloustnoe village, the right tributary Goloustnaya River, riverside,13 Aug 2012, D.A. Krivenko 29607 (IRK).

\section{CAPRIFOLIACEAE}

Linnaea borealis $\mathrm{L}$.

$2 n=32$, CHN. Russia, Republic of Buryatiya, Pribaikal'skii Raion, $3,5 \mathrm{~km} \mathrm{SW}$ from the mouth of the Turka River, vicinity of Turka village, $461 \mathrm{~m}, 52^{\circ} 54^{\prime} 59^{\prime \prime} \mathrm{N}, 108^{\circ} 11^{\prime} 03^{\prime \prime} \mathrm{E}$, clusterberry-peatmoss forest, $25 \mathrm{Jul}$ 2011, D.A. Krivenko 23271 (IRK).

\section{CARYOPHYLLACEAE}

Stellaria media (L.) Vill.

$2 n=$ ca. 40, CHN. Russia, Sverdlovskaya Oblast', Ekaterinburg city, territory of Institute of Plant \& Animal Ecology, Ural Branch of the Russian Academy of Sciences, old building, 56 49'20" N, $060^{\circ} 19^{\prime} 38^{\prime \prime}$ E, ruderal community along the asphalt walkway, 7 Sep 2011, O.S. Dymshakova 21109 (IRK).

\section{CHENOPODIACEAE}

\section{Corispermum sibiricum Iljin}

$2 n=18$, CHN. Russia, Republic of Sakha (Yakutia), Suntarskii Raion, right bank of the Vilyui River, $21 \mathrm{~km} \mathrm{SW}$ of the Suntar village, $2.7 \mathrm{~km} \mathrm{SW}$ of the Agdary village, $600 \mathrm{~m}$ above the mouth of the Arga-Diyeli River, $106 \mathrm{~m}, 61^{\circ} 59^{\prime} 03^{\prime \prime} \mathrm{N} 117^{\circ} 25^{\prime} 18^{\prime \prime}$ E, riverside, 26 Aug 2012, D.A. Krivenko 29606 (IRK).

\section{CLUSIACEAE}

Hypericum attenuatum Choisy

$2 n=16$, CHN. Russia, Irkutskaya Oblast', Irkutskii Raion, vicinity of Bol'shoe Goloustnoe village, delta Goloustnaya River, 52 $03^{\prime} 24^{\prime \prime} \mathrm{N}, 105^{\circ} 24^{\prime} 39^{\prime \prime} \mathrm{E}$, meadow steppe, 15 Aug 2012, D.A. Krivenko 29634 (IRK).

\section{CYPERACEAE}

** Carex pamirica subsp. dichroa (Freyn) T.V. Egorova

$2 n=$ ca. $32-34$, CHN. Russia, Republic of Buryatiya, Pribaikal'skii Raion, Baikal Lake, vicinity of Gremyachinsk village, $411 \mathrm{~m}, 52^{\circ} 47^{\prime} 46^{\prime \prime} \mathrm{N}, 107^{\circ} 58^{\prime} 44^{\prime \prime} \mathrm{E}$, sandy shore, $22 \mathrm{Jul}$ 2011, D.A. Krivenko 20658 (IRK).

\section{EPHEDRACEAE}

Ephedra monosperma C.A. Mey.

$2 n=14$, CHN. Russia, Irkutskaya Oblast', Nizhneudinskii Raion, Uda riverhead, left bank, near the Bogatyrskie caves, edge forest, 22 Jul 2011, T.V. Krivenko 29512 (IRK) [Fig. 3D].

\section{FABACEAE}

Astragalus frigidus (L.) A. Gray

$* * * 2 n=16+0-1 \mathrm{~B}, \mathrm{CHN}$. Russia, Irkutskaya Oblast', Nizhneilimskii Raion, $57 \mathrm{~km}$ NE of the Novaya Igirma urban village, Bol'shaya Yalyka River, about $17 \mathrm{~km}$ upstream from the river mouth, $488 \mathrm{~m}, 57^{\circ} 30^{\prime} 56^{\prime \prime} \mathrm{N}, 104^{\circ} 33^{\prime} 06^{\prime \prime} \mathrm{E}$, birch-spruce-willow-grass-sedgepeatmoss-greenmoss forest, 15 Aug 2012, S.G. Kazanovsky 24684 (IRK) [Fig. 3A].

Lespedeza davurica (Laxm.) Schindl.

$2 n=42$, CNH. Russia, Zabaikal'skii Krai, Aginskii Buryatskii District, Aginskii Raion, Nozhii Lake, $15 \mathrm{~km} \mathrm{~S}$ of the Tsokto-Khangul village, petrophytic steppe, 24 Sep 2001, G.N. Zinov'yeva 29510 (IRK) [Fig. 3E].

Melilotus albus Medik.

$2 n=16$, CNH. Russia, Zabaikal'skii Krai, vicinity of Chita city, sedge-wormwood steppe, 14 Sep 2006, G.N. Zinov'yeva 29509 (IRK).

Oxytropis varlakovii Serg.

$2 n=32$, CNH. Russia, Zabaikal'skii Krai, Akshinskii Raion, Khalanda Lake shore, 18 Jul 2007, O.D. Chernova 29507 (IRK) [Fig. 3F].

Thermopsis lanceolata $\mathrm{R}$. Br.

$2 n=18$, CHN. Russia, Zabaikal'skii Krai, Ononskii Raion, Zun-Torei Lake, feather grass steppe, 19 Jul 2000, G.N. Zinov'yeva 29503 (IRK); Russia, Zabaikal'skii Krai, Gazimuro-Zavodskii Raion, vicinity of Gazimurskii Zavod village, meadow steppe, 9 Aug 2006, O.D. Chernova 29506 (IRK) [Fig. 3G].

Vicia cracca $\mathrm{L}$

$2 n=24$, CHN. Russia, Zabaikal'skii Krai, vicinity of Chita city, on the banks of the Chita River, thicket shrubs, $30 \mathrm{Jul}$ 2007, G.N. Zinov'yeva 29511 (IRK).

\section{IRIDACEAE}

Iris biglumis Vahl

$2 n=40$, CHN. Russia, Republic of Buryatiya, Tarbagataiskii Raion, right bank of the Selenga River, northern outskirts of the Saratovka village, $505 \mathrm{~m}, 51^{\circ} 39^{\prime} 29^{\prime \prime} \mathrm{N}, 107^{\circ} 25^{\prime} 30^{\prime \prime} \mathrm{E}$, forb-grass meadow steppe, 27 Jul 2011, D.A. Krivenko 19870 (IRK).

Iris laevigata Fisch. ex Fisch. \& C.A. Mey

$2 n=32$, CHN. Russia, Republic of Buryatiya, Kabanskii Raion, Rechka Vydrinaya station, left bank of the Vydrinaya River, $200 \mathrm{~m}$ of the mouth of river, $51^{\circ} 28^{\prime} 56^{\prime \prime} \mathrm{N}, 104^{\circ} 53^{\prime} 23^{\prime \prime} \mathrm{E}$, swamp between of the railway embankment and the pebbly shaft shore Baikal Lake, 26 Aug 2010, Yu.N. Pochinchik 24372 (IRK); Russia, Republic of Buryatiya, Barguzinskii Raion, Svyatoi Nos Peninsula, coast Baikal Lake, $459 \mathrm{~m}, 53^{\circ} 35^{\prime} 51^{\prime \prime} \mathrm{N}, 108^{\circ} 52^{\prime} 37^{\prime \prime} \mathrm{E}$, shore lakes on the road, 26 Jul 2011, A.V. Verkhozina 23573 (IRK). 
Iris ruthenica Ker Gawl.

$2 n=$ ca. 80, CHN. Russia, Altaiskii Krai, Smolenskii Raion, Smolenskii pass, NE of the Smolenskoe village, $680 \mathrm{~m}, 52^{\circ} 21^{\prime} 09^{\prime \prime} \mathrm{N}$, $085^{\circ} 06^{\prime} 22^{\prime \prime} \mathrm{E}$, pine plantations forest, 22 Jun 2012 , D.A. Krivenko 29574 (IRK); Russia, Republic of Altai, Ongudaiskii Raion, Seminskii pass, 3-4 km E of the Chuiskii tract, subalpine meadow, 26 Jun 2012, D.A. Krivenko 29575 (IRK).

\section{LILIACEAE}

Tulipa biebersteiniana Schult. \& Schult.f.

$2 n=24$, CHN. Russia, Republic of Bashkortostan, Kumertauskii
Raion, near Tumanchino settlement, riverside of Belaya River, steppe slopes, 8 May 2007, N.A. Kutlunina \& A.Yu. Belyaev 556693 (SVER); Russia, Orenburgskaya Oblast', Gaiskii Raion, 5 km SW of railway Halilovo station, Guberlinskii Uplands, steppe, 6 May 2006, N.A. Kutlunina \& A.Yu. Belyaev 06-05-06 (UFU); Russia, Orenburgskaya Oblast', the railway Guberlya station of the Novotroitsk city, Guberlinskii Uplands, steppe, 7 May 2006, N.A. Kutlunina \& A.Yu. Belyaev 506719 (SVER); Russia, Orenburgskaya Oblast', Kuvandikskii Raion, near Kuvandik town, riverside of Sakmara River, floodplain forest, 6 May 2007, N.A. Kutlunina \& A.Yu. Belyaev 506722 (SVER); Russia, Volgogradskaya Oblast', Sredneahtubinskii Raion, riverside
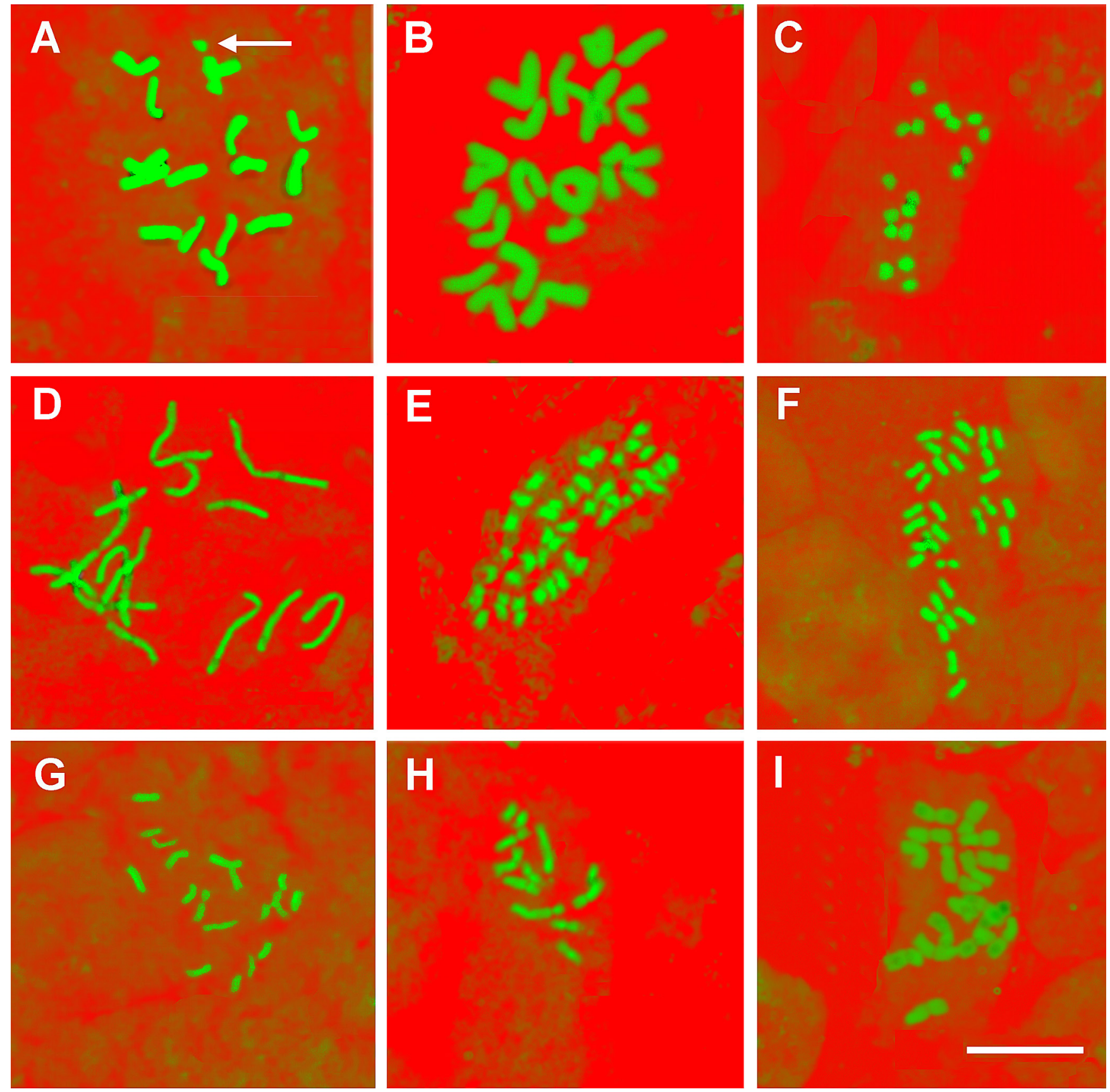

Fig. 3. A, Astragalus frigidus, $2 n=16+1 \mathrm{~B}$ (marked by arrow); B, Cypripedium guttatum, $2 n=20 ; \mathbf{C}$, Daphne mezereum, $2 n=18 ; \mathbf{D}$, Ephedra monosperma, $2 n=14 ; \mathbf{E}$, Lespedeza davurica, $2 n=42 ; \mathbf{F}$, Oxytropis varlakovii, $2 n=32 ; \mathbf{G}$, Thermopsis lanceolata, $2 n=18 ; \mathbf{H}$, Tragopogon trachycarpus, $2 n=12 ; \mathbf{I}$, Tridactylina kirilowii, $2 n=18$. - All of them mitotic counts. Scale bar $=2 \mu \mathrm{m}$. 
of Ahtuba River, floodplain forest, 18 Apr 2007, N.A. Kutlunina \& M.I. Zerebtsova 18-04-07 (UFU); Russia, Volgogradskaya Oblast', Kamyshinskii Raion, Nature Park Sherbakovkskii, rare oak forest, in depressions, 20 Apr 2007, N.A. Kutlunina \& A.Yu. Belyaev 20-04-07 (UFU).

$* * * 2 n=36$, CHN. Russia, Orenburgskaya Oblast', Guiskii Raion, $6 \mathrm{~km}$ SW from railway Halilovo station, floodplain of Bol'shaya Kayala River, 6 May 2006, N.A. Kutlunina \& A.Yu. Belyaev 06-05-06 (UFU).

Tulipa patens Agardh. ex Schult. \& Schult. f.

$2 n=24$, CHN. Russia, Orenburgskaya Oblast', Gaiskii Raion, $6 \mathrm{~km}$ SW from railway Halilovo station, Guberlinskii Uplands, tops of hills, rocky steppe, 6 May 2006, N.A. Kutlunina \& A.Yu. Belyaev 06-05-06 (UFU).

Tulipa riparia Knjaz., Kulikov \& E.g., Philippov

$2 n=36$, CHN. Russia, Chelyabinskaya Oblast', Ashinskii Raion, railway $1738 \mathrm{~km}$ station, riverside of Sim River, wet meadow, 24 May 2006, N.A. Kutlunina \& A.Yu. Belyaev 24-05-06 (UFU); Russia, Chelyabinskaya Oblast', Ashinskii Raion, near B'yanka settlement, bank of the Maloyuz stream, floodplain forest, 24 May 2006, N.A. Kutlunina \& A.Yu. Belyaev 506711 (SVER); Russia, Chelyabinskaya Oblast', Ashinskii Raion, to S from Sim city, bank of the Kuryak River near the confluence to the Sim River, wet meadow, 25 May 2006, N.A. Kutlunina \& A.Yu. Belyaev 25-05-06 (UFU).

\section{Tulipa scythica Klokov \& Zoz}

$2 n=24$, CHN. Russia, Orenburgskaya Oblast', Kuvandikskii Raion, along the route from Kuvandik town to Mednogorsk town, steppe, 19 Apr 2005, P.V. Kulikov 19-04-05 (UFU); Russia, Volgogradskaya Oblast', Leninskii Raion, near Leninsk settlement, steppe, 18 Apr 2007, N.A. Kutlunina \& M.I. Zerebtsova 18-04-07 (UFU); Russia, Volgogradskaya Oblast', Pallasovskii Raion, neighborhood of Buluhta Lake, steppe, 20 Apr 2007, N.A. Kutlunina \& M.I. Zerebtsova 20-04-07 (UFU).

\section{ONAGRACEAE}

Circaea alpina $\mathrm{L}$.

$2 n=22$, CHN. Russia, Republic of Altai, Ongudaiskii Raion, left bank of the Aigulak River, near the mouth, $1149 \mathrm{~m}, 50^{\circ} 12^{\prime} 42^{\prime \prime} \mathrm{N}$, $087^{\circ} 14^{\prime} 45^{\prime \prime} \mathrm{E}$, on the rocks covered with moss, 28 Jul 2012, D.A. Krivenko 29572 (IRK).

\section{Epilobium fastigiato-ramosum Nakai}

$2 n=36$, CHN. Russia, Irkutskaya Oblast', Ust'-Ordynskii Buryatskii District, Osinskii Raion, vicinity of Obusa village, $448 \mathrm{~m}$,

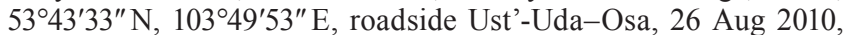
A.V. Verkhozina 27773 (IRK).

\section{ORCHIDACEAE}

Cypripedium guttatum Sw.

$2 n=20$, CHN. Russia, Republic of Buryatiya, Tunkinskii Raion, vicinity of Zun-Murino village, near the base of Institute of Plant Physiology \& Biochemistry, Siberian Branch of the Russian Academy of Sciences, $768 \mathrm{~m}, 51^{\circ} 43^{\prime} 21^{\prime \prime} \mathrm{N}, 102^{\circ} 53^{\prime} 45^{\prime \prime} \mathrm{E}$, pine-birch-alderrhododendron forest, 31 Jul 2011, S.G. Kazanovsky \& D.A. Krivenko 19885 (IRK) [Fig. 3B].

Goodyera repens (L.) R. Br.

$2 n=30$, CHN. Russia, Republic of Buryatiya, Tunkinskii Raion, vicinity of Zun-Murino village, near the base of Institute of Plant Physiology \& Biochemistry, Siberian Branch of the Russian Academy of Sciences, $768 \mathrm{~m}, 51^{\circ} 43^{\prime} 21^{\prime \prime} \mathrm{N}, 102^{\circ} 53^{\prime} 45^{\prime \prime} \mathrm{E}$, pine-birch-alderrhododendron forest, $30 \mathrm{Jul}$ 2011, S.G. Kazanovsky \& D.A. Krivenko 19796 (IRK).
Gymnadenia conopsea (L.) R. Br.

$2 n=40$, CHN. Russia, Republic of Buryatiya, Tunkinskii Raion, vicinity of Zun-Murino village, near the base of Institute of Plant Physiology \& Biochemistry, Siberian Branch of the Russian Academy of Sciences, $766 \mathrm{~m}, 51^{\circ} 43^{\prime} 21^{\prime \prime} \mathrm{N}, 102^{\circ} 53^{\prime} 38^{\prime \prime} \mathrm{E}$, pine-rhododendronforbs-lichen forest, $31 \mathrm{Jul}$ 2011, S.G. Kazanovsky \& D.A. Krivenko 20013 (IRK).

Neottianthe cucullata (L.) Schltr.

$2 n=42$, CHN. Russia, Republic of Buryatiya, Tunkinskii Raion, vicinity of Zun-Murino village, near the base of Institute of Plant Physiology \& Biochemistry, Siberian Branch of the Russian Academy of Sciences, $799 \mathrm{~m}, 51^{\circ} 43^{\prime} 17^{\prime \prime} \mathrm{N}, 102^{\circ} 53^{\prime} 40^{\prime \prime} \mathrm{E}$, pine rhododendron forbs forest, $31 \mathrm{Jul}$ 2011, S.G. Kazanovsky \& D.A. Krivenko 20021 (IRK).

\section{PLANTAGINACEAE}

Plantago depressa Willd.

$2 n=12$, CHN. Russia, Zabaikal'skii Krai, Tungiro-Olekminskii Raion, 69th km of trails Mogocha-Tupik (Geologov settlement), Burkha River, 736 m, 5414'36" N, 120 07'32" E, Larch-shrub (willow, birch scrub) forest, roadside, $12 \mathrm{Jul}$ 2011, S.G. Kazanovsky 20781 (IRK); Russia, Republic of Buryatiya, Tunkinskii Raion, vicinity of Zun-Murino village, base of Institute of Plant Physiology \& Biochemistry, Siberian Branch of the Russian Academy of Sciences, right bank of the Khyr-Gorkhon River, 704 m, 51 $43^{\prime} 15^{\prime \prime} \mathrm{N}, 102^{\circ} 53^{\prime} 37^{\prime \prime} \mathrm{E}$, grass-forb meadow, 31 Jul 2011, D.A. Krivenko 20014 (IRK).

Plantago major L.

$2 n=12$, CHN. Russia, Sverdlovskaya Oblast', Ekaterinburg city, territory of Institute of Plant \& Animal Ecology, Ural Branch of the Russian Academy of Sciences, old building, 56 $49^{\prime} 20^{\prime \prime} \mathrm{N}$, $060^{\circ} 19^{\prime} 38^{\prime \prime} \mathrm{E}$, ruderal community along the asphalt walkway, 7 Sep 2011, O.S. Dymshakova 29598 (IRK).

\section{POACEAE}

Melica turczaninowiana Ohwi

$2 n=18$, CHN. Russia, Zabaikal'skii Krai, vicinity of Chita city, Titova Sopka hill, south-eastern slope, near top, $52^{\circ} 04^{\prime} 26^{\prime \prime} \mathrm{N}$, $113^{\circ} 26^{\prime} 49^{\prime \prime} \mathrm{E}$, pine-larch forest, 13 Aug 2008, Ye.A. Bondarevich 22402 (IRK)

Milium alexeenkoi (Tzvelev) Tzvelev

$2 n=14$, CNH. Russia, Republic of Dagestan, Derbent city, along the trail up from Naryn-Kala fortress, 24 May 2009, M.P. Rayko \& V.V. Kotseruba 29597 (IRK, LE).

Phragmites flavescens (Custer) Hegetschw.

$2 n=48$, CHN. Kazakhstan, Pavlodarskaya Oblast', Bayanaul'skii Raion, Birzhankol' Lake, 391 m, 50 49'04" N, 075²0'24" E, in water, 28 Jun 2013, D.A. Krivenko 29642 (IRK).

Spodiopogon sibiricus Trin.

$2 n=40$, CHN. Russia, Zabaikal'skii Krai, Aginskii Buryatskii District, Aginskii Raion, $19 \mathrm{~km} \mathrm{~S}$ of the Aginskoye village, apricotelm thicket, on a steep rocky steppe slopes of southern exposure, $50^{\circ} 55^{\prime} 55^{\prime \prime} \mathrm{N}, 1^{\circ} 4^{\circ} 32^{\prime} 15^{\prime \prime} \mathrm{E}, 24$ Aug 2011, Ye.A. Bondarevich 22399 (IRK).

\section{POLEMONIACEAE}

Collomia linearis Nutt.

$2 n=16$, CHN. Russia, Irkutskaya Oblast', Zalarinskii Raion, vicinity of Tungui village, base of Institute of Plant Physiology \& Biochemistry, Siberian Branch of the Russian Academy of Sciences, $53^{\circ} 33^{\prime} 52^{\prime \prime} \mathrm{N}, 102^{\circ} 35^{\prime} 24^{\prime \prime} \mathrm{E}$, production field, soybean crops, $19 \mathrm{Jul}$ 2012, N.V. Dorofeev 24475 (IRK). 
Polemonium caeruleum L.

$2 n=18$, CHN. Russia, Republic of Altai, Turochakskii Raion, right bank of the Biya River, vicinity of Novotroitskoe village, $526 \mathrm{~m}$, $51^{\circ} 54^{\prime} 18^{\prime \prime} \mathrm{N}, 087^{\circ} 08^{\prime} 47^{\prime \prime} \mathrm{E}$, glade among the thickets of willow shrubs, 24 Jun 2012, D.A. Krivenko 29658 (IRK).

\section{RANUNCULACEAE}

**Aconitum sukaczevii Steinb.

$2 n=16$, CHN. Russia, Irkutskaya Oblast', Slyudyanskii Raion, vicinity of Slyudyanka town, valley of the Slyudyanka River, right bank, $594 \mathrm{~m}, 51^{\circ} 37^{\prime} 35^{\prime \prime} \mathrm{N}, 103^{\circ} 40^{\prime} 15^{\prime \prime} \mathrm{E}$, afforested steep valley sides, with rocky outcrops, 29 Jul 2011, S.G. Kazanovsky 19410 (IRK).

Ranunculus chinensis Bunge

$* * * 2 n=16+0-4 \mathrm{~B}, \mathrm{CHN}$. Russia, Zabaikal'skii Krai, Mogochinskii Raion, Pokrovka village, Amur River, 334 m, 5320'36" N, $121^{\circ} 31^{\prime} 00^{\prime \prime} \mathrm{E}$, sand-pebble shore of the river, $7 \mathrm{Jul} 2011$, Ye.S. Prelovskaya \& S.G. Kazanovsky 20959 (IRK).

\section{ROSACEAE}

Coluria geoides (Pall.) Ledeb.

$2 n=14$, CHN. Russia, Altaiskii Krai, Zmeinogorskii Raion, vicinity of Zmeinogorsk town, mountain steppe slope, 19 Jun 2012, D.A. Krivenko 29576 (IRK).

\section{SCROPHULARIACEAE}

Veronica prostrata L.

$2 n=16$, CHN. Russia, Irkutskaya Oblast', Ust'-Udinskii Raion, vicinity of Lobagai village, $468 \mathrm{~m}, 53^{\circ} 57^{\prime} 13^{\prime \prime} \mathrm{N}, 103^{\circ} 19^{\prime} 35^{\prime \prime} \mathrm{E}$, steppe slope, 29 Aug 2008, A.V. Verkhozina, S.G. Kazanovsky \& O.A. Chernyshova 27830 (IRK).

\section{SOLANACEAE}

Hyoscyamus niger $\mathrm{L}$.

$2 n=34$, CHN. Russia, Republic of Altai, Ust'-Kanskii Raion, vicinity of Chernyi Anui settlement, right bank of the Anui River, 699 m, 53²3'18" N, 08441'17" E, roadside, 6 Jul 2012, D. A. Krivenko 29628 (IRK)

\section{THYMELAEACEAE}

Daphne mezereum L.

$2 n=18$, CHN. Russia, Republic of Buryatiya, Kabanskii Raion, right bank of the Vydrinaya River, $541 \mathrm{~m}, 51^{\circ} 23^{\prime} 21^{\prime \prime} \mathrm{N}, 104^{\circ} 54^{\prime} 22^{\prime \prime} \mathrm{E}$, valley spruce-poplar-herb forest, 10 Aug 2011, S.G. Kazanovsky 21127 (IRK) [Fig. 3C].

\section{VIOLACEAE}

Viola rupestris F.W. Schmidt

$2 n=20$, CHN. Russia, Irkutskaya Oblast', Irkutskii Raion, vicinity of Bol'shoe Goloustnoe village, crease of Goloustnaya River, $52^{\circ} 02^{\prime} 14^{\prime \prime} \mathrm{N}, 105^{\circ} 27^{\prime} 07^{\prime \prime} \mathrm{E}$, on stony placers, 2 Aug 2011, D.A. Krivenko 24499 (IRK).

Viola selkirkii Pursh ex Goldie

$2 n=24$, CHN. Russia, Irkutskaya Oblast', Slyudyanskii Raion, vicinity of Slyudyanka city, valley of the Slyudyanka River, right bank, $594 \mathrm{~m}, 5^{\circ} 37^{\prime} 35^{\prime \prime} \mathrm{N}, 103^{\circ} 40^{\prime} 15^{\prime \prime} \mathrm{E}$, afforested steep valley sides, with rocky outcrops, 29 Jul 2011, S.G. Kazanovsky \& D.A. Krivenko 19469 (IRK).

\section{Maria N. Lomonosova}

Central Siberian Botanical Garden, Siberian Branch of the

Russian Academy of Science, Novosibirsk 630090, Russia;

mlomonosova@mail.ru

$\boldsymbol{\nabla}$ New chromosome number (cytotype) for the species.

* First chromosome count from Siberia.

$\Delta$ First chromosome count from Sakha (Yakutia) Republic.

** First chromosome count from Kalmykia Republic.

The research was supported by the Russian Foundation for Basic Research (grants no. 11-04-00123, 12-04-00746).

\section{AMARANTHACEAE}

Amaranthus retroflexus L.

$2 n=34$, CHN. Russia, Kalmykia Republic, Maloderbetovskii region, ecoton zone of the dried-up reservoir Barmantsak, solonchak, $47^{\circ} 58^{\prime} \mathrm{N}, 44^{\circ} 41^{\prime} \mathrm{E}, 28$ Sep 2012, M. Lomonosova $937 a$ (NS).

\section{ASTERACEAE}

Carduus crispus L.

** $2 n=18$, CHN. Russia, Kalmykia Republic, Tselinnyi region, $30 \mathrm{~km}$ of Volgograd, salt meadow, $46^{\circ} 40^{\prime} \mathrm{N}, 4^{\circ} 18^{\prime} \mathrm{E}, 30$ Sep 2012, M. Lomonosova 959 a (NS).

Centaurea diffusa Lam

** $2 n=18$, CHN. Russia, Kalmykia Republic, Sarpinskii region, vicinities of Arshan-Zelmen reservoir, roadside, $47^{\circ} 35^{\prime} \mathrm{N}, 44^{\circ} 35^{\prime} \mathrm{E}$, 28 Sep 2012, M. Lomonosova 941 a (NS).

Lactuca serriola $\mathrm{L}$.

$2 n=18$, CHN. Russia, Novosibirskaya Oblast', Karasuk, waste ground, 13 Sep 2012, M. Lomonosova 925 a (NS).

Lactuca tatarica (L.) C.A. Mey.

** $2 n=18$, CHN. Russia, Kalmykia Republic, Priyutninskii region, $32 \mathrm{~km}$ of Elista to Priyutnoe, dried Phragmites community, 46 $51^{\prime} \mathrm{N}, 4^{\circ} 55^{\prime}$ E, 29 Sep 2012, M. Lomonosova 952a (NS); ibid., Sarpinskii region, between Sadovyi village and Arshan-Zelmen reservoir, roadside, $47^{\circ} 41^{\prime} \mathrm{N}, 44^{\circ} 31^{\prime} \mathrm{E}$, $30 \mathrm{Sep} 2012$, M. Lomonosova 961 (NS).

Saussurea amara (L.) DC.

$2 n=26$, CHN. Russia, Novosibirskaya Oblast', Karasuk, waste ground, 13 Sep 2012, M. Lomonosova $925 b$ (NS).

Tragopogon podolicus (DC.) S.A. Nikitin

$2 n=12$, CHN. Russia, Novosibirskaya Oblast', Karasuk, waste ground, 13 Sep 2012, M. Lomonosova 924a, M. Lomonosova 925c (NS).

Xanthium strumarium L.

$2 n=36$, CHN. Russia, Novosibirskaya Oblast', Karasuk, roadside near the railway station, 13 Sep 2012, M. Lomonosova $926 a$ (NS).

\section{BRASSICACEAE}

Lepidium densiflorum Schrad.

$\boldsymbol{\nabla} * 2 n=16$, CHN. Russia, Sakha (Yakutia) Republic, Khangalasskii ulus, vicinities of Bulgunnyakhtakh village, stony southern slope, $61^{\circ} 17^{\prime} \mathrm{N}, 128^{\circ} 35^{\prime} \mathrm{E}, 25$ Aug 2012, M. Lomonosova \& E. Nikolin 859 (NS).

Thlaspi arvense $\mathrm{L}$.

$\Delta 2 n=14$, CHN. Russia, Yakutia Republic, Namskii ulus, vicinities of Betyuntsy village, oats field, $62^{\circ} 48^{\prime} \mathrm{N}, 129^{\circ} 37^{\prime} \mathrm{E}, 20 \mathrm{Aug}$ 2012, M. Lomonosova \& E. Nikolin $791 a$ (NS). 


\section{CARYOPHYLLACEAE}

Dianthus versicolor Fisch. \& Link

$\boldsymbol{\nabla} \boldsymbol{\Delta} 2 n=60$, CHN. Russia, Sakha (Yakutia) Republic, Namskii ulus, vicinities of Betyuntsy village, edge of birch forest, $62^{\circ} 48^{\prime} \mathrm{N}$, $129^{\circ} 37^{\prime}$ E, 20 Aug 2012, M. Lomonosova \& E. Nikolin 793 (NS).

\section{CHENOPODIACEAE}

Atriplex micrantha C.A. Mey.

$* * 2 n=36$, CHN. Russia, Kalmykia Republic, Priyutninskii region, Priyutnoe, roadside, 29 Sep 2012, M. Lomonosova 949a. (NS); ibid., Sarpinskii region, vicinities of Arshan-Zelmen reservoir, desert steppe, $47^{\circ} 35^{\prime} \mathrm{N}, 44^{\circ} 35^{\prime} \mathrm{E}, 28$ Sep 2012, M. Lomonosova 940 (NS)

\section{Atriplex patens (Litv.) Iljin}

$2 n=36$, CHN. Russia, Novosibirskaya Oblast', Karasuk, salt meadow, 13 Sep 2012, M. Lomonosova 922 (NS).

Atriplex prostrata Boucher \& DC.

$* * 2 n=18$, CHN. Russia, Kalmykia Republic, Tselinnyi region, in vicinity of Khar-Bulak village, "Alone populus" spring, solonchak in a hollow, 29 Sep 2012, M. Lomonosova 954a (NS); ibid., $30 \mathrm{~km}$ of the tract from Elista to Volgograd, 29 Sep 2012, M. Lomonosova 959 b (NS).

Atriplex sagittata Borkh.

$2 n=18$, CHN. Russia, Novosibirskaya Oblast', Karasuk, waste ground, 13 Sep 2012, M. Lomonosova $924 b$ (NS).

Atriplex tatarica $\mathrm{L}$.

** $2 n=18$, CHN. Russia, Kalmykia Republic, Maloderbetovskii region, ecoton zone of the dried-up reservoir Barmantsak, solonchak, $47^{\circ} 58^{\prime} \mathrm{N}, 4^{\circ} 41^{\prime} \mathrm{E}, 28$ Sep 2012, M. Lomonosova $937 b$ (NS); ibid., Sarpinskii region, vicinities of Arshan-Zelmen reservoir, roadside, $47^{\circ} 35^{\prime} \mathrm{N}, 44^{\circ} 35^{\prime} \mathrm{E}, 28 \mathrm{Sep} 2012$, M. Lomonosova $941 b$ (NS).

Bassia hyssopifolia (Pall.) Kuntze

$2 n=18$, CHN. Russia, Kalmykia Republic, Tselinnyi region, $30 \mathrm{~km}$ from Tselinnoe to Volgograd, $46^{\circ} 40^{\prime} \mathrm{N}, 4^{\circ} 18^{\prime} \mathrm{E}$, 30 Sep 2012 , M. Lomonosova 958 a (NS).

Bassia scoparia (L.) A.J. Scott (三Kochia scoparia (L.) Schrad.)

$* * 2 n=18$, CHN. Russia, Kalmykia Republic, Tselinnyi region, $30 \mathrm{~km}$ of Tselinnoe to Volgograd, $46^{\circ} 40^{\prime} \mathrm{N}, 44^{\circ} 18^{\prime} \mathrm{E}, 30 \mathrm{Sep}$ 2012, M. Lomonosova $958 b$ (NS); Russia, Khakasia Republic, UstAbakanskii region, vicinities of Solnechnoe village, steppe, 18 Oct 2012, F. Kudrov 963 (NS).

Caroxylon dendroides (Pall.) Tzvelev

$2 n=18$, CHN. Russia, Kalmykia Republic, Priyutninskii region, north shore of Manych-Gudilo reservoir, steppe, $46^{\circ} 05^{\prime} \mathrm{N}, 43^{\circ} 27^{\prime} \mathrm{E}$, 29 Sep 2012, M. Lomonosova 947 (NS).

Chenopodium acerifolium Andrz.

$\Delta 2 n=18$, CHN. Russia, Sakha (Yakutia) Republic, MeginoKangalasskii ulus, Kolymskii tract, at the bridge over Suola river, sandy river bank, $62^{\circ} 05^{\prime} \mathrm{N}, 130^{\circ} 09^{\prime} \mathrm{E}, 28$ Aug 2012 , M. Lomonosova \& E. Nikolin $876(\mathrm{NS})$.

Chenopodium album $\mathrm{L}$.

** $2 n=54$, CHN. Russia, Kalmykia Republic, Priyutninskii region, Priyutnoe, waste ground, 29 Sep 2012, M. Lomonosova 949 a (NS).

Chenopodium borbasii Murr

$* * 2 n=54$, CHN. Russia, Kalmykia Republic, Elista, flower bed, 30 Sep 2012, M. Lomonosova 957 (NS).
Chenopodium ficifolium $\mathrm{Sm}$.

$2 n=18$, CHN. Russia, Sakha (Yakutia) Republic, Namskii ulus, vicinities of Betyuntsy village, oats field, $62^{\circ} 48^{\prime} \mathrm{N}, 129^{\circ} 37^{\prime} \mathrm{E}, 20$ Aug 2012, M. Lomonosova \& E. Nikolin $791 b$ (NS).

Chenopodium strictum Roth

$* * 2 n=36$, CHN. Russia, Novosibirskaya Oblast', Karasuk, waste ground, 13 Sep 2012, M. Lomonosova 925d (NS); Russia, Kalmykia Republic, Elista, "Chess City", roadside, 30 Sep 2012, M. Lomonosova 960 (NS).

\section{Chenopodium suecicum Murr}

$2 n=18$, CHN. Russia, Novosibirskaya Oblast', Novosibirsk, Akademgorodok, roadside, 11 Sep 2012, M. Lomonosova 965 (NS).

Kali tragus (L.) Scop. ( $\equiv$ Salsola tragus L.)

$* * 2 n=36$, CHN. Russia, Novosibirskaya Oblast', Karasuk, roadside near the railway station, 13 Sep 2012, M. Lomonosova $926 b$ (NS); Russia, Kalmykia Republic, Elista, roadside, 29 Sep 2012, M. Lomonosova 944 (NS); ibid., Tselinnyi region, $30 \mathrm{~km}$ of Elista to Volgograd, roadside, $46^{\circ} 41^{\prime} \mathrm{N}, 4^{\circ} 19^{\prime} \mathrm{E}, 30 \mathrm{Sep} 2012$, M. Lomonosova $958 c$ (NS).

Oxybasis chenopodioides (L.) S. Fuentes, Uotila \& Borsch (三 Chenopodium chenopodioides (L.) Aellen)

$* * 2 n=18$, CHN. Russia, Kalmykia Republic, Priyutninskii region, $32 \mathrm{~km}$ of the tract from Elista to Priyutnoe, dried Phragmites community, $46^{\circ} 11^{\prime} \mathrm{N}, 43^{\circ} 55^{\prime} \mathrm{E}$, 29 Sep 2012, M. Lomonosova $952 b$ (NS).

Oxybasis glauca (L.) S. Fuentes, Uotila \& Borsch

(三 Chenopodium glaucum L.)

$2 n=18$; Russia, Novosibirskaya Oblast', Karasuk, waste ground, 13 Sep 2012, M. Lomonosova 925e (NS).

Oxybasis urbica (L.) S. Fuentes, Uotila \& Borsch

( $\equiv$ Chenopodium urbicum $\mathrm{L}$.)

$* * 2 n=18$, CHN. Russia, Kalmykia Republic, Elista, roadside, 29 Sep 2012, M. Lomonosova 944 (NS); ibid., Priyutnoe, waste ground, 29 Sep 2012, M. Lomonosova 949c (NS).

Salicornia perennans Willd.

$2 n=18$, CHN. Russia, Kalmykia Republic, Priyutninskii region, $32 \mathrm{~km}$ of Elista to Priyutnoe, dried Phragmites community, 46 $51^{\prime} \mathrm{N}$, 435'ㄹ, 29 Sep 2012, M. Lomonosova 952c (NS); Russia, Sakha (Yakutia) Republic, Namskii tract near Zhatay village, salt meadow with Puccinellia, 20 Aug 2012, M. Lomonosova \& E. Nikolin 783 (NS); ibid., Namskii ulus, vicinities of Khamagatta village, solonchak, $62^{\circ} 40^{\prime} \mathrm{N}, 1^{\circ} 9^{\circ} 41^{\prime} \mathrm{E}, 20$ Aug 2012, M. Lomonosova \& E. Nikolin 790 (NS); ibid., Namskii ulus, vicinities of Khatyryk village, solonchak, $63^{\circ} 01^{\prime} \mathrm{N}, 129^{\circ} 31^{\prime} \mathrm{E}, 20$ Aug 2012, M. Lomonosova \& E. Nikolin 796 (NS).

Salsola soda $\mathrm{L}$

** $2 n=18$, CHN. Russia, Kalmykia Republic, Maloderbetovskii region, ecoton zone of the dried-up reservoir Barmantsak, solonchak, $47^{\circ} 58^{\prime} \mathrm{N}, 44^{\circ} 41^{\prime} \mathrm{E}, 28$ Sep 2012, M. Lomonosova 938 a (NS).

Sedobassia sedoides (Pall.) Freitag \& G. Kadereit

(三 Bassia sedoides (Pall.) Asch.)

* $2 n=18$, CHN. Russia, Novosibirskaya Oblast', Karasuk, waste ground, $13 \mathrm{Sep}$ 2012, M. Lomonosova 924c (NS).

Suaeda acuminata (C.A. Mey.) Moq.

$2 n=18$, CHN. Russia, Kalmykia Republic, Maloderbetovskii region, ecoton zone of the dried-up reservoir Barmantsak, solonchak, $47^{\circ} 58^{\prime} \mathrm{N}, 4^{\circ} 41^{\prime} \mathrm{E}, 28$ Sep 2012, M. Lomonosova $938 b$ (NS); ibid., 
Priyutninskii region, $32 \mathrm{~km}$ of Elista to Priyutnoe, roadside, $46^{\circ} 11^{\prime} \mathrm{N}$, 435'르, 29 Sep 2012, M. Lomonosova 951 (NS).

Suaeda corniculata (C.A. Mey.) Bunge subsp. corniculata $2 n=54$, CHN. Russia, Khakasia Republic, Ust-Abakanskii region, Krasnoe lake, salt meadow, 20 Sep 2012, F. Kudrov 964 a (NS).

Suaeda salsa (L.) Pall.

**2n $=36$, CHN. Russia, Kalmykia Republic, Maloderbetovskii region, ecoton zone of the dried-up reservoir Barmantsak, solonchak, $4^{\circ} 58^{\prime} \mathrm{N}, 4^{\circ} 41^{\prime} \mathrm{E}, 28$ Sep 2012, M. Lomonosova 937c (NS); ibid., Priyutninskii region, northern coast of the reservoir Manych-Gudilo, on the bottom of dried lake, $46^{\circ} 05^{\prime} \mathrm{N}, 43^{\circ} 27^{\prime} \mathrm{E}, 29 \mathrm{Sep} 2012, M$. Lomonosova 948 (NS); ibid., Tselinnyi region, vicinities of Khar-Bulak village, "Alone populus" spring, solonchak in a hollow, 29 Sep 2012, M. Lomonosova $954 b$ (NS); ibid., Tselinnyi region, $30 \mathrm{~km}$ from Elista to Volgograd, salt meadow, $46^{\circ} 40^{\prime} \mathrm{N}, 44^{\circ} 18^{\prime} \mathrm{E}$, 30 Sep $2012, M$. Lomonosova 958d, M. Lomonosova 959c (NS); Russia, Volgogradskaya Oblast', southern suburb of Volgograd, solonchak along the road, 30 Sep 2012, M. Lomonosova 962 (NS).

Suaeda sibirica Lomon. \& Freitag

$2 n=72$, CHN. Russia, Khakasia Republic, Ust-Abakanskii region, Krasnoe lake, solonchak, 20 Sep 2012, F. Kudrov $964 b$ (NS).

\section{PLANTAGINACEAE}

Plantago cornuti Gouan

$2 n=12$, CHN. Russia, Novosibirskaya Oblast', Karasuk, waste ground, 13 Sep 2012, M. Lomonosova $925 f$ (NS)

\section{ROSACEAE}

Potentilla paradoxa Nutt. ex Torr. \& Gray

$2 n=42$, CHN. Russia, Novosibirskaya Oblast', Karasuk, waste ground, 13 Sep 2012, M. Lomonosova $925 g$ (NS).

Nina S. Probatova, ${ }^{1 *}$ Sergey G. Kazanovsky, ${ }^{2}$ Elvira G. Rudyka, ${ }^{1}$ Vitaly P. Seledets ${ }^{3}$ \& Svetlana V. Ovchinnikova ${ }^{4}$

1 Institute of Biology \& Soil Science, Far East Branch of the Russian Academy of Sciences, 159 Stoletya Prospect, 690022, Vladivostok, Russia

2 Siberian Institute of Plant Physiology \& Biochemistry, Siberian Branch of the Russian Academy of Sciences, 132 Lermontov Str., 664033, Irkutsk, Russia

3 Pacific Institute of Geography, Far East Branch of the Russian Academy of Sciences, 7 Radio Str., 690041, Vladivostok, Russia

4 Central Siberian Botanical Garden, Siberian Branch of the Russian Academy of Sciences, 101 Zolotodolinskaya Str., 630090, Novosibirsk, Russia

* Author for correspondence: probatova@ibss.dvo.ru

* First chromosome count for the species.

** New chromosome number (cytotype) for the species.

This study was supported by grants no. 11-04-00240 (to N.S. Probatova), 11-04-01167 (to V.S. Shneyer), 12-04-01586 (to A.V. Verkhozina) from the Russian Fund for Basic Research (RFBR), and by Interdisciplinary integration project $\mathrm{Nr}$. 17, from the Siberian Branch of the Russian Academy of Sciences.

\section{ADOXACEAE}

Adoxa moschatellina L.

$2 n=36$, CHN. Russia, Far East, Primorskii Krai, MuravëvAmurskii Peninsula, Vladivostok, near Sanatornaya, forest part of the Botanical Garden-Institute, Far East Branch of the Russian Academy of Science, forest on lower part of the slope, 12 May 2013, Semeikin 12348 (VLA).

\section{ALISMATACEAE}

Alisma plantago-aquatica $\mathrm{L}$.

$2 n=14$, CHN. Russia, East Siberia, Irkutskaya Oblast', Ust'Kutskii Raion, 8.5 km WNW of Yarakta oilmans' settlement, left riverside of the Yarakta River, $442 \mathrm{~m}$, near the bridge, roadside, in the puddle, 9 Aug 2012, Kazanovsky 12369 (VLA, IRK).

\section{ALLIACEAE}

Allium condensatum Turcz.

$2 n=16$, CHN. Russia, Far East, Primorskii Krai, Peter the Great Bay, Klykova Island, 30 Jun 2000, Prozorova 8179 (VLA).

\section{APIACEAE}

Kitagawia baicalensis (Redowsky ex Willd.) Pimenov

$2 n=22$, CHN. Russia, East Siberia, Republic of Buryatiya, Tunkinskii Raion, near Zun-Murino village, scientific station of the Siberian Institute of Plant Physiology \& Biochemistry, the KhyrGorkhon River, 704 m, sandy slope, 25 Sep 2012, Kazanovsky 12419 (VLA, IRK)

Oenanthe javanica (Blume) DC.

$2 n=22$, CHN. Russia, Far East, Primorskii Krai, MuravëvAmurskii Peninsula, near Okeanskaya, the Botanical Garden Institute of the Far East Branch of the Russian Academy of Sciences, at the artificial pond, 27 Oct 2012, Volynets \& Semeikin 12214 (VLA).

$2 n=44$, CHN. Russia, Far East, Primorskii Krai, Peter the Great Bay, Reineke Island, moist depression near the settlement, 26 Aug 1999, Probatova \& Seledets 7895 (VLA).

\section{ARACEAE}

Arisaema amurense Maxim.

$2 n=56$, CHN. Russia, Far East, Primorskii Krai, Partizanskii Raion, the shore of Vostok Bay, flood-lands of the Volchanka River, 19 May 2013, Nechaev 12349 (VLA).

\section{ASTERACEAE}

Arctium minus (Hill.) Bernh.

$2 n=36$, CHN. Russia, Far East, Primorskii Krai, Peter the Great Bay, Elena Island (close to Russkii Island), as a weed on the waste place near the abandoned building, 19 Sep 1997, Probatova \& Seledets 7554 (VLA).

Artemisia scoparia Waldst. \& Kit.

$2 n=16$, CHN. Russia, East Siberia, Republic of Buryatiya, Tunkinskii Raion, right riverside of the Margasan River, $5 \mathrm{~km} \mathrm{~S}$ of Margasan locality, steep rocky SE slope, 25 Sep 2012, Kazanovsky 12408 (VLA, IRK).

Artemisia tanacetifolia $\mathrm{L}$.

$2 n=54$, CHN. Russia, East Siberia, Republic of Buryatiya, Tunkinskii Raion, right riverside of the Margasan River, $5 \mathrm{~km} \mathrm{~S}$ of Margasan locality, Pinus and Betula forest with grasses and various herbs, 25 Sep 2012, Kazanovsky 12412 (VLA, IRK).

Glebionis segetum Fourr.

$2 n=18$, CHN. Russia, Far East, Primorskii Krai, MuravëvAmurskii Peninsula, Vladivostok, between Akademgorodok and the factory "Zarya", roadside, 27 Oct 2012, Semeikin 12217 (VLA).

Lapsana communis L.

$2 n=14$, CHN. Russia, North Caucasus, Krasnodarskii Krai, Abinskii Raion, in vicinity of Erivanskaya stanitsa, forest edge, 5 Sep 2009, Probatova \& Seledets 11510 (VLA). 
Solidago dahurica Kitag.

$2 n=18$, CHN. Russia, East Siberia, Irkutskaya Oblast', Tulunskii Raion, $2 \mathrm{~km}$ WNW of the railway station Shuba, $502 \mathrm{~m}$, the Betula forest edge, meadow community, 16 Aug 2012, Kazanovsky 12372 (VLA, IRK).

Youngia tenuifolia (Willd.) Babc. \& Stebbins

$2 n=10$, CHN. Russia, East Siberia, Republic of Buryatiya, Tunkinskii Raion, in vicinity of Zun-Murino village, scientific station of the Siberian Institute of Plant Physiology \& Biochemistry, the Khyr-Gorkhon River, 704 m, sandy slope, 25 Sep 2012, Kazanovsky 12410 (VLA, IRK).

\section{BORAGINACEAE}

Brachybotrys paridiformis Maxim. ex Oliv.

$2 n=24$, CHN. Russia, Far East, Primorskii Krai, MuravëvAmurskii Peninsula, near Okeanskaya, forest part of the Botanical Garden-Institute of the Far East Branch of Russian Academy of Sciences, W slope, coniferous-broadleaved forest, 16 Jun 2012, Volynets 12139 (VLA)

\section{BRASSICACEAE}

Arabis pendula $\mathrm{L}$.

$2 n=16$, CHN. Russia, Far East, Primorskii Krai, Mikhailovskii Raion, W outskirts of Novoshakhtinskii town, as a weed in a vegetable garden, 9 Sep 2012, Lapenko 12355 (VLA).

Erysimum cheiranthoides L.

$2 n=16$, CHN. Russia, East Siberia, Irkutskaya Oblast', Ust'Kutskii Raion, $18 \mathrm{~km}$ SSE of Yarakta oilmans' settlement, the Nizhnya Tunguska River, near the bridge, $436 \mathrm{~m}$, ruderal plant community, 8 Aug 2012, Kazanovsky 12385 (VLA, IRK).

Rorippa palustris (L.) Besser

$2 n=16$, CHN. Russia, Far East, Primorskii Krai, Shkotovskii Raion, near Shkotovo settlement, stony embankment at the electric line, by the rivulet, 24 Aug 2013, Nechaev 12424 (VLA).

\section{CARYOPHYLLACEAE}

Dianthus versicolor Fisch. ex Link

$2 n=30$, CHN. Russia, East Siberia, Republic of Buryatiya, Tunkinskii Raion, right riverside of the Margasan River, $5 \mathrm{~km} \mathrm{~S}$ of Margasan locality, steep rocky SE slope, 25 Sep 2012, Kazanovsky 12362 (VLA, IRK).

Gastrolychnis brachypetala (Hornem.) Tolm. \& Kozhanch.

** $2 n=24$, CHN. Russia, East Siberia, Republic of Buryatiya, Okinskii Raion, near Samarta settlement, East Sayan Mts., Kitoiskii Range, the upper course of the Kitoi River, 1890 m, in shrub community (Caragana jubata) along the riverside, 12 Jul 2008, Kazanovsky 12387 (VLA, IRK).

Stellaria crassifolia Ehrh.

$2 n=26$, CHN. Russia, East Siberia, Zabaikalskii Krai, Mogochinskii Raion, Mogocha settlement, the riverside of the Amazar River, $610 \mathrm{~m}$, Carex and grasses moist meadow, 6 Jul 2011, Kazanovsky 12375 (VLA, IRK).

\section{Stellaria graminea $\mathrm{L}$}

$2 n=26$, CHN. Russia, East Siberia, Irkutskaya Oblast', Kuitunskii Raion, outskirts of Kuitun town, 535 m, near the car-service station, meadow by the road, 16 Aug 2012, Kazanovsky 12409 (VLA, IRK).

Stellaria longifolia Muhl. ex Willd.

$2 n=26$, CHN. Russia, East Siberia, Irkutskaya Oblast', Katangskii Raion, $9.5 \mathrm{~km}$ SE of Bur village, $435 \mathrm{~m}$, Picea and Larix waterlogged forest with shrubs and green mosses, 11 Aug 2012, Kazanovsky 12416 (VLA, IRK).

\section{CONVALLARIACEAE}

Maianthemum dilatatum (Alph. Wood) A. Nelson \& J.F. Macbr.

$2 n=54$, CHN. Russia, Far East, Primorskii Krai, Peter the Great Bay, Russkii Island, $2 \mathrm{~km} \mathrm{NW}$ of the Cape Vyatlina, forest edge, 24 Sep 1999, Rudyka 7998 (VLA).

\section{CONVOLVULACEAE}

Calystegia dahurica Choisy

$2 n=22$, CHN. Russia, East Siberia, Republic of Buryatiya, Tunkinskii Raion, right riverside of the Margasan River, $5 \mathrm{~km} \mathrm{~S}$ of Margasan locality, steep rocky SE slope, 25 Sep 2012, Kazanovsky 12370 (VLA, IRK).

\section{CYPERACEAE}

Carex siderosticta Hance

$2 n=24$, CHN. Russia, Far East, Primorskii Krai, Peter the Great Bay, Russkii Island, Shiguino, forest on the slope, 22 Aug 2011, Rudyka 11904 (VLA).

\section{GERANIACEAE}

Geranium pratense L.

$2 n=28$, CHN. Russia, East Siberia, Irkutskaya Oblast', Ust'Kutskii Raion, $18 \mathrm{~km}$ SSE of Yarakta oilmans' settlement, the Nizhnaya Tunguska River, near the bridge, $436 \mathrm{~m}$, the riverside shrubs community, 8 Aug 2012, Kazanovsky 12403 (VLA, IRK).

\section{IRIDACEAE}

Iris setosa Pall. ex Link

$2 n=38$, CHN. Russia, Far East, Primorskii Krai, Peter the Great Bay, Russkii Island, Shiguino, meadow at the bottom of a slope, 22 Aug 2011, Rudyka 11882 (VLA).

\section{LAMIACEAE}

Clinopodium chinense Kuntze

$2 n=20$, CHN. Russia, Far East, Primorskii Krai, MuravëvAmurskii Peninsula, Okeanskii Range, $5 \mathrm{~km}$ NNW of Lazurnyi settlement, 312 m, SE slope, Artemisia gmelinii community, 21 Oct 2004, Probatova \& Seledets 9555 (VLA).

Dracocephalum nutans L.

$2 n=10$, CHN. Russia, East Siberia, Irkutskaya Oblast', Nukutskii Raion, near Zarechnyi settlement, steppe community with grasses and various herbs, 11 Jun 2009, Prelovskaya 12396 (VLA, IRK).

Elsholtzia ciliata (Thunb.) Hyl.

$2 n=16$, CHN. Russia, West Siberia, Republic of Altai, Chemal'skii Raion, Chemal village, right riverside of the Katun' River, opposite Patmos Island, $415 \mathrm{~m}$, the edge of Pinus and Betula forest, at the road, 31 Aug 2012, Kazanovsky 12380 (VLA, IRK).

Lagopsis supina (Steph.) Ikonn.-Gal.

$2 n=18$, CHN. Russia, East Siberia, Republic of Buryatiya, Dzhidinskii Raion, Petropavlovka village, $675 \mathrm{~m}$, steppe community with grasses and various herbs, 13 Jul 2009, Kazanovsky 12404 (VLA, IRK).

Leonurus glaucescens Bunge

$2 n=18$, CHN. Russia, West Siberia, Republic of Altai, Chemal'skii Raion, Chemal village, right riverside of the Katun' River, between Patmos Island and Chemal'skaya hydroelectric power station, 435 m, forest on the rocky slope, 30 Aug 2012, Kazanovsky 12388 (VLA, IRK). 
Mosla dianthera (Buch.-Ham. ex Roxb.) Maxim.

$2 n=18$, CHN. Russia, Far East, Primorskii Krai, Nakhodkinskii administrative raion, S outskirts of Livadia settlement, the bank of a small reservoir near Livadiiskoe Lake, 22 Sep 2012, Lapenko 12346 (VLA).

Nepeta sibirica $\mathrm{L}$.

$2 n=18$, CHN. Russia, West Siberia, Republic of Altai, Ongudaiskii Raion, in vicinity of Ongudai settlement, $958 \mathrm{~m}$, stony S slope with steppe vegetation, 30 Aug 2012, Kazanovsky 12377 (VLA, IRK).

Schizonepeta multifida (L.) Briq.

$2 n=12$, CHN. Russia, East Siberia, Republic of Buryatiya, Tunkinskii Raion, right riverside of the Margasan River, $5 \mathrm{~km} \mathrm{~S}$ of Margasan locality, steep rocky SE slope, 25 Sep 2012, Kazanovsky 12357 (VLA, IRK).

\section{LILIACEAE}

Lilium pilosiusculum (Freyn) Miscz.

$2 n=24$, CHN. Russia, East Siberia, Irkutskaya Oblast', Slyudyanskii Raion, 61-st km of the Kultukskii tract (road), near Staraya Angasolka village, $788 \mathrm{~m}$, the edge of Larix and Betula forest with various herbs, 24 Sep 2012, Kazanovsky 12417 (VLA, IRK).

\section{LOBELIACEAE}

Lobelia sessilifolia Lamb.

$2 n=28, C H N$. Russia, Far East, Primorskii Krai, Partizanskii Raion, between the coast of Vostok Bay and Dushkino settlement, the maritime plain, grassy bog, 31 Aug 2013, Nechaev 12422 (VLA).

\section{LYTHRACEAE}

Lythrum salicaria $\mathrm{L}$.

$2 n=30$, CHN. Russia, Far East, Primorskii Krai, MuravëvAmurskii Peninsula, in vicinity of Sedanka railway station, forest edge, at the wayside ditch, 27 Aug 2013, Kapustina 12423 (VLA).

\section{PAPAVERACEAE}

Papaver olchonense Peschkova

$2 n=28$, CHN. Russia, East Siberia, Irkutskaya Oblast', Ol'khonskii Raion, Cape Zunduk, right riverside of the Zunduk River, 564 m, stony NE slope with steppe vegetation, 27 Jun 2012, Kazanovsky 12411 (VLA, IRK).

\section{POACEAE}

Bromopsis inermis (Leyss.) Holub

$2 n=56$, CHN. Russia, East Siberia, Irkutskaya Oblast', Nizhneilimskii Raion, $5 \mathrm{~km}$ E of Novaya Iguirma town, $346 \mathrm{~m}$, at the road, 15 Aug 2012, Kazanovsky 12407 (VLA, IRK).

Calamagrostis obtusata Trin.

$2 n=28$, CHN. Russia, West Siberia, Republic of Altai, Ongudaiskii Raion, Chuiskii tract, Seminskii Pass, 1720 m, light Pinus sibirica forest with various herbs, 30 Aug 2012, Kazanovsky 12420 (VLA, IRK)

Digitaria asiatica (Ohwi) Tzvelev

$2 n=18$, CHN. Russia, Far East, Primorskii Krai, Mikhailovskii Raion, W outskirts of Novoshakhtinskii town, as a weed in a vegetable garden, 29 Aug 2012, Lapenko 12350 (VLA); Russia, Far East, Primorskii Krai, Ussuriyskii Raion, left riverside of the Razdol'naya (Suifun) River, near Utësnoe village, 18 Jun 1998, coll. unknown 7744 (VLA); Russia, Far East, Primorskii Krai, Peter the Great Bay, Elena Island (near Russkii Island), along the path to Larionova Cape, 19 Sep 1997, Probatova \& Seledets 7556 (VLA).
Digitaria ischaemum (Schreb.) Muhl.

$2 n=36$, CHN. Russia, Far East, Primorskii Krai, Mikhailovskii Raion, W outskirts of Novoshakhtinskii town, the Ozërnaya Pad' railway station, on the railroad embankment, 9 Sep 2012, Lapenko 12352 (VLA).

Koeleria tokiensis Domin

$2 n=14$, CHN. Russia, Far East, Primorskii Krai, Peter the Great Bay, Lissii Island, 11 Sep 2002, Nechaev 8906 (VLA).

Poa alpina L.

$2 n=28$, CHN. Russia, East Siberia, Republic of Buryatiya, Okinskii Raion, near Samarta settlement, East Sayan Mts., Kitoiskii Range, the upper course of the Kitoi River, 1889 m, meadow in the shrub community (Caragana jubata, Rhododendron, Salix spp.), 28 Aug 2010, Kazanovsky 12344 (VLA, IRK).

Puccinellia andersonii Swallen

$* * 2 n=42$, CHN. Russia, Far East, Primorskii Krai, Terneiskii Raion, Sikhote-Alinskii biosphere reserve, locality Abrek, near Yaponskoe Lake, on maritime rocks, 6 Aug 2003, Nesterova 9665 (VLA).

Puccinellia interior T.J. Sørensen

$* 2 n=28$, CHN. Russia, Far East, Primorskii Krai, Dal'negorskii Raion, outskirts of Dal'negorsk town, the spring Tretii, rubbly littered plots, 17 Jul 1984, Probatova, Seledets \& al. 6407 (VLA).

Puccinellia paupercula Fernald \& Weath.

$* 2 n=42$, CHN. Russia, Far East, Primorskii Krai, Peter the Great Bay, Popova Island, the Stark Strait, plots of the shore covered by high tide, 22 Sep 1979, Probatova \& Seledets 5524 (VLA).

Puccinellia schischkinii Tzvelev

$* 2 n=14$, CHN. Russia, Far East, Primorskii Krai, Vladivostok city, near Pervaya Rechka railway station, waste area at the railroad embankment, 28 Sep 1974, Probatova \& Seledets 3940 (VLA).

This specimen have been misidentified as "P. tenuissima" in Bot. Zhurn. 62(8): 1149. 1977.

Torreyochloa natans (Kom.) G.L. Church

$2 n=14$, CHN. Russia, Far East, Sakhalin, Korsakovskii Raion, $2 \mathrm{~km}$ SE of Ozërsk settlement, the Komissarovka River, near the mouth, low river bank, 5 Oct 1980, Probatova \& Seledets 5826 (VLA).

\section{POLYGALACEAE}

Polygala tenuifolia Willd.

$2 n=34$, CHN. Russia, East Siberia, Republic of Buryatiya, Dzhidinskii Raion, $10 \mathrm{~km} \mathrm{SW}$ of Dyrestui village, left riverside of the Dzhida River, 596 m, steppe with Ulmus pumila, 24 Jul 2009, Kazanovsky 12398 (VLA, IRK).

\section{PRIMULACEAE}

Lysimachia volkovae Prob.

$* 2 n=24$, CHN. Russia, Far East, Primorskii Krai, Vladivostok city, Vtoraya Rechka, Russkaya Str., as a weed on the flower-bed, near the building, 29 Jul 2013, Probatova \& Seledets 12418 (VLA).

\section{RANUNCULACEAE}

Aquilegia oxysepala Trautv. \& C.A. Mey.

$2 n=16$, CHN. Russia, Far East, Primorskii Krai, Khassanskii Raion, $40 \mathrm{~km}$ W of Razdol'noe settlement, vicinity of Kravtsovka village, forest on the slope, 3 May 1980, Probatova \& Seledets 5693 (VLA).

Aquilegia turczaninovii Kamelin \& Gubanov

$2 n=16$, CHN. Russia, East Siberia, Zabaikal'skii Krai, Borzinskii Raion, mountain-steppe massif Adun-Chelon, 1019 m, 
on the rocks, in the fissure filled with melkozem-humus soil, $15 \mathrm{Jul}$ 2010, Kazanovsky 12360 (VLA, IRK).

\section{ROSACEAE}

Geum canadense Jacq.

$2 n=42$, CHN. U.S.A., Texas, Addison (near Dallas), nature forest park, 3 Apr 2005, Rudyka 9740 (VLA).

Potentilla fragarioides $\mathrm{L}$.

$2 n=14$, CHN. Russia, Primorskii Krai, Vladivostok city, Kariernaya Str., on the slope, 21 Jun 2007, Kapustina 10730 (VLA).

Potentilla norvegica $\mathrm{L}$.

$2 n=56$, CHN. Russia, Primorskii Krai, Nadezhdinskii Raion, in vicinity of Taëzhnoe settlement, near country place "Al'batros", roadside, 28 Jun 1998, Probatova \& Seledets 7824 (VLA).

Potentilla recta $\mathrm{L}$.

$2 n=28$, CHN. Russia, North Caucasus, Krasnodarskii Krai, Novorossiiskii Raion, in vicinity of stanitsa Raevskaya, Volchii Vorota Pass, rubbly placer of marl, 3 Sep 2009, Probatova \& Seledets 12072 (VLA).

Potentilla tanacetifolia Willd. ex D.F.K. Schltdl.

$2 n=28$, CHN. Russia, East Siberia, Republic of Buryatiya, Tunkinskii Raion, right riverside of the Margasan River, $5 \mathrm{~km} \mathrm{~S}$ of Margasan locality, steep rocky SE slope, 25 Sep 2012, Kazanovsky 12338 (VLA, IRK).

\section{SCROPHULARIACEAE}

Veronica linariifolia Pall. ex Link

$2 n=34$, CHN. Russia, East Siberia, Republic of Buryatiya, Tunkinskii Raion, right riverside of the Margasan River, $5 \mathrm{~km} \mathrm{~S}$ of Margasan locality, steep rocky SE slope, 25 Sep 2012, Kazanovsky 12374 (VLA, IRK).

\section{SOLANACEAE}

Solanum nigrum $\mathrm{L}$.

$2 n=72$, CHN. Russia, Primorskii Krai, Mikhailovskii Raion, W outskirts of Novoshakhtinskii town, as a weed in the vegetable garden, 21 Sep 2010, Lapenko 12339 (VLA).

\section{VALERIANACEAE}

Patrinia rupestris (Pall.) Juss.

$2 n=22$, CHN. Russia, East Siberia, Republic of Buryatiya, Tunkinskii Raion, right riverside of the Margasan River, $5 \mathrm{~km} \mathrm{~S}$ of Margasan locality, steep rocky SE slope, 25 Sep 2012, Kazanovsky 12371 (VLA, IRK).

\section{Nina S. Probatova, ${ }^{1 *}$ Alla V. Verkhozina, ${ }^{2}$ Elvira G. Rudyka, ${ }^{1}$ \& Denis A. Krivenko ${ }^{2}$}

1 Institute of Biology \& Soil Science, Far East Branch of the Russian Academy of Sciences, 159 Stoletya Prospect, 690022, Vladivostok, Russia

2 Siberian Institute of Plant Physiology \& Biochemistry, Siberian Branch of the Russian Academy of Sciences, 132 Lermontov Str., 664033, Irkutsk, Russia

* Author for correspondence: probatova@ibss.dvo.ru

* First chromosome count for the species.

This study was supported by grants no. 11-04-00240 (to N.S. Probatova), 12-04-01586 (to A.V. Verkhozina), 12-04-31151 (to D.A. Krivenko) from the Russian Fund for Basic Research (RFBR), and by
Interdisciplinary integration project Nr. 17, from the Siberian Branch of the Russian Academy of Sciences.

\section{ALLIACEAE}

Allium ramosum $\mathrm{L}$.

$2 n=32$, CHN. Russia, Far East, Amurskaya Oblast', Arkharinskii Raion, Arkhara town, 10 Sep 2012, Kudrin 12330 (VLA).

\section{ASTERACEAE}

Erigeron elongatus Ledeb.

$2 n=18$, CHN. Russia, East Siberia, Irkutskaya Oblast', Katangskii Raion, $16.5 \mathrm{~km} \mathrm{~S}$ of Bur settlement, $460 \mathrm{~m}$, winter road in Larix and Picea forest with Vaccinium myrtillus and green mosses, on moist sabulous soil, near waterlogged plot, 10 Aug 2012, Kazanovsky \& Verkhozina 12373 (VLA, IRK).

Galatella dahurica DC.

$2 n=18$, CHN. Russia, East Siberia, Republic of Sakha-Yakutia, Suntarskii Rayon, left riverside of the Vilyui River, opposite the mouth of the Arga-Dieli River (right tributary of the Vilyui River), $21 \mathrm{~km} \mathrm{SW}$ of Suntar settlement, $3.3 \mathrm{~km} \mathrm{SSW}$ of Agdary village, $111 \mathrm{~m}$, meadow with steppe elements on S slope to the Vilyui River, 27 Aug 2012, Krivenko 12414 (VLA, IRK).

\section{Lactuca serriola L.}

$2 n=18$, CHN. Russia, Far East, Amurskaya Oblast', Arkharinskii Raion, Arkhara town, waste ground, roadside, 23 Aug 2012, Kudrin 12323 (VLA).

Matricaria perforata Mérat

$2 n=36$, CHN. Russia, East Siberia, Irkutskaya Oblast', Shelekhovskii Raion, in vicinity of Smolenshchina settlement, $437 \mathrm{~m}$, the edge of a field, 1 Sep 2006, Verkhozina \& Tolstonogova 12384 (VLA, IRK).

Pterocypsela indica (L.) C. Shih

$2 n=18$, CHN. Russia, Far East, Amurskaya Oblast', Arkharinskii Raion, Khinganskii nature reserve, Antonovskoe forestry, square 11, 23 Aug 2012, Kudrin 12324 (VLA).

Tanacetum vulgare L.

$2 n=18$, CHN. Russia, East Siberia, Republic of Sakha-Yakutia, Suntarskii Rayon, left riverside of the Vilyui River, opposite the mouth of the Arga-Dieli River (right tributary of the Vilyui River), $21 \mathrm{~km} \mathrm{SW}$ of Suntar settlement, $3.3 \mathrm{~km} \mathrm{SSW}$ of Agdary village, $111 \mathrm{~m}$, meadow with steppe elements on S slope to the Vilyui River, 27 Aug 2012, Krivenko 12415 (VLA, IRK).

\section{BORAGINACEAE}

Echium vulgare L.

$2 n=16$, CHN. Russia, East Siberia, Irkutskaya Oblast', Nizhneilimskii Raion, near Khrebtovaya settlement, 592 m, meadow at the road, 14 Aug 2012, Verkhozina 12359 (VLA, IRK).

Lappula lenensis Popov ex Ovczinnikova

$* 2 n=24$, CHN. Russia, East Siberia, Republic of Sakha-Yakutia, Suntarskii Raion, right riverside of the Vilyui River, $21 \mathrm{~km} \mathrm{SW}$ of Suntar settlement, $2.7 \mathrm{~km} \mathrm{SW}$ of Agdary village, $600 \mathrm{~m}$ above the mouth of the Arga-Dieli River, $106 \mathrm{~m}$, the bank of the Vilyui River, 26 Aug 2012, Verkhozina 12395 (VLA, IRK).

\section{BRASSICACEAE}

Bunias orientalis $\mathrm{L}$.

$2 n=14$, CHN. Russia, East Siberia, Irkutskaya Oblast', SE outskirts of Taishet town, near 5th Tzelinnaya Str., ruderal plots at the road, 7 Aug 2012, Verkhozina \& al., 12391 (VLA, IRK). 
Descurainia sophia (L.) Webb ex Prantl

$2 n=14$, CHN. Russia, East Siberia, Irkutskaya Oblast', Katangskii Raion, $9.5 \mathrm{~km}$ SE of Bur settlement, the pipe line, plug place, on dumped rockfill, 12 Aug 2012, Verkhozina \& Kazanovsky 12382 (VLA, IRK)

Lepidium latifolium $\mathrm{L}$.

$2 n=24$, CHN. Russia, East Siberia, Irkutskaya Oblast', SE outskirts of Taishet town, along the railway lines, 7 Aug 2012, Verkhozina \& al. 12390 (VLA, IRK).

\section{FABACEAE}

Astragalus frigidus (L.) A. Gray

$2 n=16+0-2 \mathrm{~B}, \mathrm{CHN}$. Russia, East Siberia, Republic of Buryatiya, Tunkinskii Rayon, the valley of the Irkut River, right riverbank, in vicinity of Moigoty village, pine forest, 11 Aug 2012, Krivenko 29593 (IRK).

Astragalus mongholicus Bunge

$2 n=16$, CHN. Russia, East Siberia, Irkutskaya Oblast', Ol'khonskii Rayon, Baikal Lake, Olkhon Island, near Shara-Nur Lake, pine forest, 15 Aug 2012, Prelovskaya \& Bondarevich 28970 (IRK).

Astragalus sericeocanus Gontsch.

$2 n=16+0-1 \mathrm{~B}, \mathrm{CHN}$. Russia, East Siberia, Republic of Buryatiya, Pribaikal'skii Rayon, $5 \mathrm{~km} \mathrm{SW}$ of the mouth of the Turka River, in vicinity of Turka village, $452 \mathrm{~m}$, sand dunes along the shore of Baikal Lake, forest edge, 24 Jul 2011, Zhmud' 23490 (IRK).

\section{CARYOPHYLLACEAE}

Lychnis chalcedonica $\mathrm{L}$.

$2 n=24$, CHN. Russia, East Siberia, Irkutskaya Oblast', Nizhneilimskii Raion, near Khrebtovaya settlement, 583 m, meadow at the road, probably on the place of the former vegetable garden, 15 Aug 2012, Kazanovsky \& Verkhozina 12402 (VLA, IRK).

\section{LAMIACEAE}

Dracocephalum thymiflorum $\mathrm{L}$.

$2 n=14$, CHN. Russia, East Siberia, Irkutskaya Oblast', SE outskirts of Taishet town, along the railway lines, 7 Aug 2012, Verkhozina \& al. 12386 (VLA, IRK).

Leonurus villosus Desf.

$2 n=18$, CHN. Russia, East Siberia, Irkutskaya Oblast', Taishet town, near the railway station, Matrossova Str., ruderal plant communities along the road, 4 Aug 2012, Verkhozina \& Kaverzina 12379 (VLA, IRK).

\section{LYTHRACEAE}

Lythrum salicaria $\mathrm{L}$

$2 n=30$, CHN. Russia, East Siberia, Irkutskaya Oblast', SE outskirts of Taishet town, 1-st Tzelinnaya Str., $313 \mathrm{~m}$, the littered slope of a road and alongside, plots with superfluous damping, 7 Aug 2012, Verkhozina \& al. 12381 (VLA, IRK).

\section{PAPAVERACEAE}

Papaver nudicaule $\mathrm{L}$.

$2 n=28$, CHN. Russia, East Siberia, Republic of Buryatiya, Barguzinskii Raion, the Baikal Lake, Svyatoi Nos Peninsula, $16.5 \mathrm{~km}$ NNW of the mouth of Barguzin River, $447 \mathrm{~m}$, dune sands, 26 Jul 2011, Verkhozina 12392 (VLA, IRK).

\section{PLANTAGINACEAE}

Plantago major L.

$2 n=12$, CHN. Russia, East Siberia, Irkutskaya Oblast',
Ust'-Kutskii Raion, 8.5 km WNW of Yarakta oilmans' settlement, left riverside of the Yarakta River, $447 \mathrm{~m}$, at the road near the bridge, overgrown rut along the bank of Yarakta River, 9 Aug 2012, Verkhozina 12421 (VLA, IRK).

\section{POACEAE}

Agrostis clavata Trin

$2 n=42$, CHN. Russia, Far East, Amurskaya Oblast', Arkharinskii Raion, Arkhara town, at the road, 20 Jul 2012, Parilova 12311 (VLA).

Digitaria asiatica Tzvelev

$2 n=18$, CHN. Russia, Far East, Khabarovskii Krai, the railway station Khabarovsk-1, as a weed on the railroad embankment, 4 Sep 2004, Probatova \& Seledets 10484 (VLA).

Setaria glareosa Petrov

* $2 n=18$, CHN. Russia, East Siberia, Irkutskaya Oblast', Katangskii Raion, $9.5 \mathrm{~km}$ SE of Bur settlement, $440 \mathrm{~m}$, the pipe line, plug place, on dumped rockfill, 12 Aug 2012, Kazanovsky \& Verkhozina 12376 (VLA, IRK).

Setaria pumila (Poir.) Roem. \& Schult. (S. glauca auct.)

$2 n=18$, CHN. Russia, Far East, Amurskaya Oblast', Arkharinskii Raion, Khinganskii nature reserve, Antonovskoe forestry, the lakeside of Klëshinskoe Lake, on the bank, 21 Aug 1982, Yashenkova 6302 (VLA).

$2 n=36$, CHN. Russia, Volga Region, Volgogradskaya Oblast', N outskirts of Volgograd city, gardening plots near GRES settlement, 2 Aug 2006, Yurtseva 10496 (VLA).

\section{PORTULACACEAE}

Portulaca oleracea $\mathrm{L}$.

$2 n=36$, CHN. Russia, Far East, Amurskaya Oblast', Arkharinskii Raion, Arkhara town, near the office of the Khinganskii nature reserve, on sandy ground, 10 Sep 2012, Kudrin 12319 (VLA).

\section{ROSACEAE}

Comarum palustre L.

$2 n=28$, CHN. Russia, East Siberia, Irkutskaya Oblast', Katangskii Raion, $2 \mathrm{~km}$ SE of Bur settlement, $412 \mathrm{~m}$, Larix and Picea forest with shrubs and green mosses, 11 Aug 2012, Verkhozina \& Kazanovsky 12401 (VLA, IRK).

Potentilla longifolia Willd. ex D.F.K. Schltdl.

$2 n=14$, CHN. Russia, East Siberia, Republic of Sakha-Yakutia, Suntarskii Raion, left riverside of the Vilyui River, opposite the mouth of the Arga-Dieli River (right tributary of the Vilyui River), $21 \mathrm{~km}$ SSW of Agdary village, $111 \mathrm{~m}$, meadow with steppe elements on S slope to the Vilyui River, 27 Aug 2012, Krivenko 12337 (VLA, IRK).

\section{Potentilla norvegica $\mathrm{L}$.}

$2 n=56$, CHN. Russia, East Siberia, Irkutskaya Oblast', Ust'Kutskii Raion, 8.5 km WNW of Yarakta oilmans' settlement, left riverside of the Yarakta River, $447 \mathrm{~m}$, at the road near the bridge, overgrown rut along the bank of Yarakta River, 9 Aug 2012, Verkhozina 12383 (VLA, IRK).

Potentilla paradoxa Nutt. ex Torr. \& Gray

$2 n=28$, CHN. Russia, East Siberia, Republic of Sakha-Yakutia, Suntarskii Raion, right riverside of the Vilyui River, $20 \mathrm{~km} \mathrm{SW}$ of Suntar settlement, $2 \mathrm{~km} \mathrm{SW}$ of Agdary village, near the mouth of the Arga-Dieli River, 106 m, sandy bank, 27 Aug 2012, Verkhozina 12413 (VLA, IRK); Russia, East Siberia, Republic of Buryatiya, Pribaikal'skii Raion, $5.3 \mathrm{~km} \mathrm{SW}$ of Turka village, $464 \mathrm{~m}$, roadside, 23 Jul 2011, Verkhozina \& Anossova 12340 (VLA, IRK). 


\section{María Santos-Vicente, ${ }^{*}$ Enrique Rico \& M. Montserrat Martínez-Ortega}

Departamento de Botánica, Universidad de Salamanca, 37007 Salamanca, Spain.

* Author for correspondence:m.santos@usal.es

* First chromosome count for the species.

- First chromosome count for an accession of the species from the referenced country.

$\nabla$ Endemic to the Iberian Peninsula.

+ Endemic to North Africa.

This study was supported by the Spanish Dirección General de Investigación (Ministerio de Ciencia y Tecnología and Ministerio de Educación y Ciencia) through the projects Flora iberica VI (REN2002-04634-C05-02; grant ref. BES-2003-2213 to M. Santos Vicente) and Flora iberica VII (CGL2005-05471-C04-03) and by the Junta de Castilla y León through project SA142A08. We greatly acknowledge this support.

\section{ASTERACEAE}

Inula helenioides DC.

* $2 n=32$, CHN. Spain, La Rioja, Lagunilla del Jubera towards Zenzano, $42^{\circ} 21^{\prime} 13^{\prime \prime} \mathrm{N}, 02^{\circ} 20^{\prime} 25^{\prime \prime} \mathrm{W}, 571 \mathrm{~m}$, slopes and roadsides, dolomites, 1 Jul 2006, A. Abad, S. Andrés-Sánchez, M.M. MartínezOrtega \& M. Santos-Vicente 758 (SALA 136037) [Fig. 4A]

$* 2 n=40$, CHN. Spain, Valladolid, San Martín de Valvení, $41^{\circ} 45^{\prime} 25^{\prime \prime} \mathrm{N}, 04^{\circ} 31^{\prime} 39^{\prime \prime} \mathrm{W}, 865 \mathrm{~m}$, nitrophilous pastures, roadside, 6 Jul 2007, L. Delgado \& M. Santos-Vicente 907 (SALA 136055) [Fig. 4B]; Spain, Soria, El Royo, Hinojosa de la Sierra, 41 ${ }^{\circ} 51^{\prime} 49^{\prime \prime} \mathrm{N}$, 02 ${ }^{\circ} 35^{\prime} 34^{\prime \prime} \mathrm{W}, 1030 \mathrm{~m}$, riverbank, calcareous soil, 23 Aug 2007, L. Delgado, C. Molina \& M. Santos-Vicente 953b (SALA 136056) [Fig. 4C].

A previously published chromosome count $(2 n=16$; Löve \& Kjellqvist, 1974) needs to be discarded since the voucher specimen (RNG) corresponds to just a few leaves that for sure cannot be identified as I. helenioides. Therefore, these are the first reliable counts for this species endemic to France and Spain.

\section{Inula langeana Beck}

$\boldsymbol{\nabla} * 2 n=32$, CHN. Spain, Burgos, Jurisdicción de San Zadornil, San Millán de San Zadornil, 42 50'29" N, 0309'14" W, 680 m, pasture in Pinus sylvestris and Quercus ilex forest, calcareous soil, $13 \mathrm{Jul}$ 2004, S. Andrés-Sánchez, J.A. Sánchez-Agudo \& M. Santos-Vicente 412 (SALA 136057) [Fig. 4D].

$\boldsymbol{\nabla} * 2 n=40$, CHN. Spain, Valladolid, Tudela de Duero, $41^{\circ} 34^{\prime} 23^{\prime \prime} \mathrm{N}, 04^{\circ} 30^{\prime} 42^{\prime \prime} \mathrm{W}, 863 \mathrm{~m}$, Quercus faginea and Pinus pinaster forest, calcareous soil, 14 Jun 2005, P. Bariego \& M. Santos-Vicente 583 (SALA 136058) [Fig. 4E].

$\boldsymbol{\nabla} * 2 n=48$, CHN. Spain, Palencia, Hontoria de Cerrato, $41^{\circ} 54^{\prime} 52^{\prime \prime} \mathrm{N}, 004^{\circ} 23^{\prime} 42^{\prime \prime} \mathrm{W}, 800 \mathrm{~m}$, base of slopes, calcareous soil, 10 Aug 2007, L. Delgado \& M. Santos-Vicente 952 (SALA 136059); Spain, Álava, Lantarón, Puentelarrá, $42^{\circ} 44^{\prime} 34^{\prime \prime} \mathrm{N}, 03^{\circ} 02^{\prime} 31^{\prime \prime} \mathrm{W}, 440 \mathrm{~m}$, Quercus ilex forest, calcareous soil, 10 Jun 2006, A. Abad, S. AndrésSánchez, M.M. Martinez-Ortega \& M. Santos-Vicente 755 (SALA

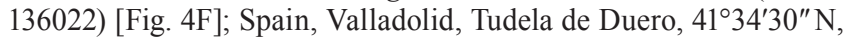
$004^{\circ} 30^{\prime} 42^{\prime \prime} \mathrm{W}, 850 \mathrm{~m}$, Quercus faginea and Pinus pinaster forest, calcareous soil, 10 Aug 2007, L. Delgado \& M. Santos-Vicente 946 (SALA 136060).

This is the first report for this endemic species, which is restricted to CN Iberian Peninsula. This taxon has been traditionally subordinated to I. conyzae (Griess.) DC., but morphological evidence supports the independence of both species (Santos-Vicente \& al., in prep.). In addition, a single cytotype has been found in I. conyzae (Santos-Vicente $\&$ al., 2009), whereas I. langeana shows three ploidy levels.
Inula maletii Maire

$+* 2 n=32$, CHN. Morocco, Fès-Boulemane, Ifrane, Tizi-nTretten, $33^{\circ} 27^{\prime} 32^{\prime \prime} \mathrm{N}, 05^{\circ} 02^{\prime} 09^{\prime \prime} \mathrm{W}, 1980 \mathrm{~m}$, clearings on Quercus ilex and Cedrus atlantica forest, calcareous soil, $10 \mathrm{Jul}$ 2006, A. Herrero \& al. 3347 (SALA 136015) [Fig. 4G]; Morocco, Tadla-Azilal, Beni Mellal, El Ksiba, 32 $30^{\prime} 36^{\prime \prime} \mathrm{N}, 06^{\circ} 00^{\prime} 04^{\prime \prime} \mathrm{W}, 1878$ m, Quercus ilex forest, calcareous soil, $5 \mathrm{Jul}$ 2006, A. Quintanar \& al. 2043 (SALA 136016); Morocco, Tadla-Azilal, Col de Tanout ou Fillal, $32^{\circ} 40^{\prime} 38^{\prime \prime} \mathrm{N}$, $05^{\circ} 27^{\prime} 17^{\prime \prime} \mathrm{W}, 2070 \mathrm{~m}$, Quercus ilex forest, calcareous soil, 6 Jul 2006, A. Quintanar \& al. 2153 (SALA 136007).

Inula maletii is a North-African endemic very narrowly distributed in the Middle Atlas of Morocco (ca. 10 populations). Inula maletii is morphologically similar to I. bifrons, but the latter species (scattered in S Europe) shows a different cytotype $(2 n=16$, diploid level; Santos-Vicente \& al., 2009).

Inula montana $\mathrm{L}$.

$2 n=16$, CHN. Spain, Palencia, Vertavillo, $41^{\circ} 48^{\prime} 58^{\prime \prime} \mathrm{N}$, $04^{\circ} 15^{\prime} 07^{\prime \prime} \mathrm{W}, 811 \mathrm{~m}$, Quercus faginea forest, calcareous soil, 20 Jun 2007, L. Delgado \& M. Santos-Vicente 882 (SALA 136061).

- $2 n=16$, CHN. Morocco, Marrakech-Tensift-Al Haouz, Oukaïmeden, $31^{\circ} 11^{\prime} 52^{\prime \prime} \mathrm{N}, 07^{\circ} 51^{\prime} 55^{\prime \prime} \mathrm{W}, 2700 \mathrm{~m}$, pastures, sandstones, 30 Jun 2006, A. Herrero \& al. 3215 (SALA 136064) [Fig. 4H].

The chromosome numbers obtained agree with those reported for Spain (Löve \& Kjellqvist, 1974), France (Guinochet \& Logeois, 1962; Delay, 1968) and Italy (D'Amato, 1971; Brullo \& al., 1977). This is the first chromosome count for an accession of I. montana from North Africa (Morocco). The species has been also reported from Algeria

Inula oculus-christi $\mathrm{L}$.

$n=16$, CHN. Bulgaria, Smolyan, Buynovsko Gorge, $41^{\circ} 38^{\prime} 30^{\prime \prime} \mathrm{N}$, $24^{\circ} 20^{\prime} 03^{\prime \prime} \mathrm{E}, 1040 \mathrm{~m}$, rocky places, calcareous soil, 2 Jun 2004, C. Navarro \& al. 4769 (SALA 136063).

This haploid chromosome number agrees with that reported by Baksay (1958) for plants collected in Hungary and it is consistent with the previously published diploid number (e.g., Strid \& Franzen, 1981; Probatova \& al., 2009), including Bulgarian counts (Kuzmanov \& Georgieva, 1977).

Inula salicina $\mathrm{L}$.

$2 n=16$, CHN. Spain, Lleida, Bellver de Cerdanya, $42^{\circ} 20^{\prime} 11^{\prime \prime} \mathrm{N}$, 0143'31" E, moist pastures, 1260 m, 1 Jul 2004, X. Giráldez, A. Guillén \& M. Santos-Vicente 404 (SALA 136062); Spain, Salamanca, Aldeacipreste, $40^{\circ} 22^{\prime} 24^{\prime \prime} \mathrm{N}, 05^{\circ} 53^{\prime} 13^{\prime \prime} \mathrm{W}, 860$ m, clearings on Fraxinus angustifolia and Quercus pyrenaica forest, siliceous soil, 8 Jun 2005, L. Delgado \& M. Santos-Vicente 572 (SALA 136045) [Fig. 4I].

Most of the chromosome counts published for this taxon agree with ours (e.g., Löve \& Kjellqvist, 1974; Lövkvist \& Hultgard, 1999). Supernumerary chromosomes have been reported in the literature for I. salicina $(2 n=16+2 B$; Capineri \& al., 1978; Ghaffari, 1987), and some exceptional reports ( $2 n=18$; Kuzmanov \& Kozucharov, 1970; Dobeš \& al., 1997) could also be explained due to the presence of two B chromosomes. Nevertheless, supernumerary chromosomes have not been observed in the Spanish populations studied.

\section{Literature cited}

Baksay, L. 1958. The chromosome numbers of Ponto-Mediterranean plant species. Ann. Hist.-Nat. Mus. Natl. Hung. 50: 121-125.

Brullo, S., Pavone, P., Terrasi, M.C. \& Zizza, A. 1977. Numeri cromosomi per la flora italiana: 299-314. Inform. Bot. Ital. 9: 57-71.

Capineri, R., D'Amato, G. \& Marchi, P. 1978. Numeri cromosomic para la flora italiana: 543-583. Inform. Bot. Ital. 10: 421-465.

D'Amato, G. 1971. Numeri cromosomi per la flora italiana: 67-80. Inform Bot. Ital. 3: 138-147.

Delay, J. 1968. Halophytes II. Inform. Annuelles Caryosyst. Cytogén. 2: $17-22$. 
Dobeš, C., Hahn, B. \& Morawetz, W. 1997. Chromosomenzahlen zur Gefäßpflanzen-Flora von Österreichs. Linzer Biol. Beitr. 29: 5-43.

Ghaffari, S.M. 1987. Chromosome studies in some flowering plants of Iran. Rev. Cytol. Biol. Vég. Botaniste 10: 3-8.

Guinochet, M. \& Logeois, A. 1962. Premières prospections caryologiques dans la flore des Alpes maritimes. Rev. Cytol. Biol. Vég. 25: 465-480.

Kuzmanov B.A. \& Georgieva S. 1977. [Report]. In: Löve, A. (ed.), IOPB Chromosome number reports LVII. Taxon 26: 443-452.

Kuzmanov, B.A. \& Kozucharov, S.I. 1970. [Report]. In: Löve, A. (ed.), IOPB chromosome number reports XXVI. Taxon 19: 264-269.

Löve, A. \& Kjellqvist, E. 1974. Cytotaxonomy of spanish plants. IV. Dicotyledons: Caesalpinaceae-Asteraceae. Lagascalia 4: 153-211.
Lövkvist, B. \& Hultgård, U.-M. 1999. Chromosome numbers in south Swedish vascular plants. Opera Bot. 137: 1-42.

Probatova, N.S., Seledets, V.P., Rudyka, E.G., Gnutikov, A.A., Kozhevnikova, Z.V. \& Barkalov, V.Y. 2009. Inula oculus-christi L. In: Marhold, K. (ed.), IAPT/IOPB chromosome data 8. 58: Taxon 58: 1285 , E12.

Santos-Vicente, M., Rico, E. \& Martínez-Ortega, M.M. 2009. Inula bifrons L., I. conyzae (Griess.) Meikle, I. ensifolia L., I. helenium L. \& I. helvetica Grauer (Asteraceae). In: Marhold, K. (ed.), IAPT/IOPB chromosome data 8. Taxon 58: 1288, E20-E21.

Strid, A. \& Franzén, R. 1981. In: Löve, A. (ed.), IOPB Chromosome number reports LXXIII. Taxon 30: 829-842.
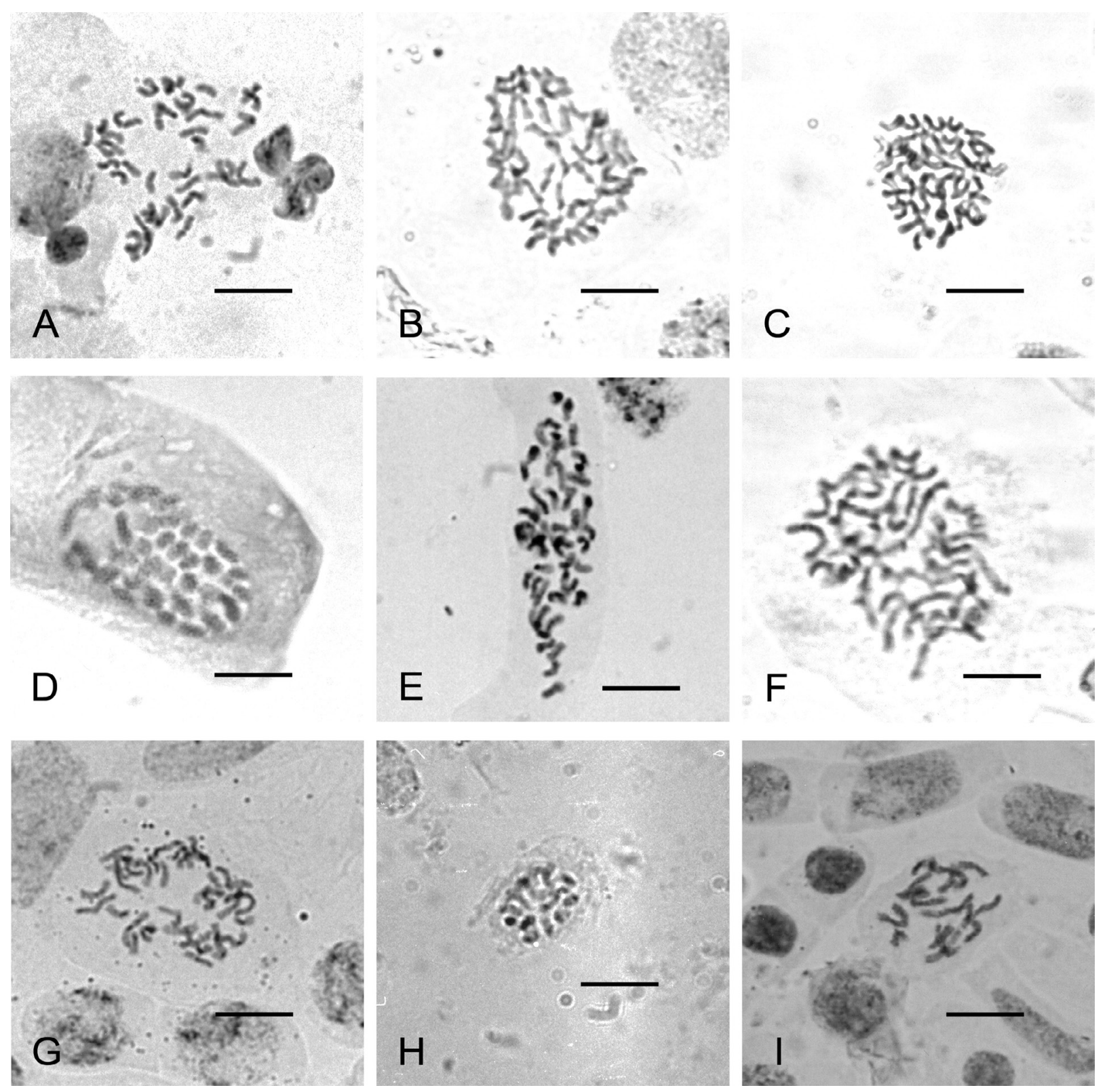

Fig. 4. A, Inula helenioides, $2 n=32$ (A. Abad, S. Andrés-Sánchez, M.M. Martínez-Ortega \& M. Santos-Vicente 758; SALA 136037); B, I. helenioides, $2 n=40$ (L. Delgado \& M. Santos-Vicente 907; SALA 136055); C, I. helenioides, $2 n=40$ (L. Delgado, C. Molina \& M. Santos-Vicente 953b; SALA 136056); D, I. langeana, $2 n=32$ (S. Andrés-Sánchez, J.A. Sánchez-Agudo \& M. Santos-Vicente 412; SALA 136057); E, I. langeana, $2 n=40$ (P. Bariego \& M. Santos-Vicente 583; SALA 136058); F, I. langeana, $2 n=48$ (A. Abad, S. Andrés-Sánchez, M.M. Martínez-Ortega \& M. Santos-Vicente 755; SALA 136022); G, I. maletii, $2 n=32$ (A. Herrero \& al. 3347; SALA 136015); H, I. montana $2 n=16$ (A. Herrero \& al. 3215 ; SALA 136064); I, I. salicina, $2 n=16$ (L. Delgado \& M. Santos-Vicente 572; SALA 136045). — All of them mitotic counts. Scale bar $=10 \mu \mathrm{m}$. 